\title{
Expedient Microwave-Assisted Synthesis of Bis(n)-lophine Analogues as Selective Butyrylcholinesterase Inhibitors: Cytotoxicity Evaluation and Molecular Modelling
}

\author{
Viktor S. Câmara, ${ }^{a}$ Ana Julia Soares, ${ }^{a}$ Brunella Biscussi, ${ }^{\oplus b}$ Ana Paula Murray, ${ }^{\circledR *, b}$ \\ Isabella A. Guedes, ${ }^{\oplus c}$ Laurent E. Dardenne, ${ }^{\oplus *, c}$ Thaís C. Ruaro, ${ }^{d}$ Aline R. Zimmer ${ }^{*, d}$ \\ and Marco A. Ceschi ${ }^{\odot} *, a$ \\ ${ }^{a}$ Instituto de Química, Universidade Federal do Rio Grande do Sul, \\ Av. Bento Gonçalves, 9500, Campus do Vale, 91501-970 Porto Alegre-RS, Brazil \\ ${ }^{b}$ Departamento de Química, Instituto de Química del Sur, \\ Consejo Nacional de Investigaciones Científicas y Técnicas (INQUISUR-CONICET), \\ Universidad Nacional del Sur, Avenida Alem, 1253, B8000CPB Bahía Blanca, Argentina \\ 'Laboratório Nacional de Computação Científica, Av. Getulio Vargas, 333, \\ 25651-075 Petrópolis-RJ, Brazil \\ 'Programa de Pós-Graduação em Ciências Farmacêuticas, Faculdade de Farmácia, \\ Universidade Federal do Rio Grande do Sul, Av. Ipiranga, 2752, 90610-000 Porto Alegre-RS, Brazil
}

In the brain of patients with chronic Alzheimer's disease (AD), the butyrylcholinesterase (BuChE) levels rise while the acetylcholinesterase (AChE) levels decrease. Therefore, development of new selective BuChE inhibitors is of vital importance. Here we present a series of bis $(n)$-lophine analogues, where two lophine derivatives are connected by a methylene chain. The bis $(n)$-lophine analogues were synthesized through one-pot four component reaction between pyridinecarboxaldehydes, $1, n$-alkanediamines, benzil, and ammonium acetate. The reactions were performed in a microwave reactor in one step for symmetrical bis $(n)$-lophines, and in two steps for unsymmetrical bis $(n)$-lophines. The compounds are strongly selective to $\mathrm{BuChE}$, since none of them inhibit AChE. All the compounds, except $\mathbf{7 a}, \mathbf{7 b}$ and $\mathbf{7 c}$, displayed potent inhibitory activity against $\mathrm{BuChE}$ at a micromolar and sub-micromolar range (half maximal inhibitory concentration $\left(\mathrm{IC}_{50}\right)$ 32.25-0.03 $\left.\mu \mathrm{M}\right)$. The enzyme kinetic and docking studies suggests that the inhibitor act as a dual binding site inhibitor, binding into the bottom of the gorge and in the peripheral anionic site (PAS) of BuChE cavity. Furthermore, in vitro studies showed that compounds $\mathbf{5 b}$ and $\mathbf{1 2 b}$ had no cytotoxic effects in kidney Vero, hepatic HepG2 and C6 astroglial cell lines.

Keywords: cholinesterase inhibitor, butyrylcholinesterase, bis(n)-lophine analogues

\section{Introduction}

The serine hydrolases acetylcholinesterase (AChE) and butyrylcholinesterase $(\mathrm{BuChE})$ are structurally related enzymes that co-regulate the metabolism of the neurotransmitter acetylcholine (ACh).

Cholinesterase inhibitors (ChEIs) are a class of drugs that have been used in the management of various human ailments, including Alzheimer's disease (AD), ${ }^{1}$ Parkinson's disease, ${ }^{2}$ glaucoma,${ }^{3,4}$ myasthenia gravis, ${ }^{5}$ Lewy bodies' disease, ${ }^{6}$ and chronic pain in elderly. ${ }^{7}$

*e-mail: apmurray@uns.edu.ar; dardenne@lncc.br; mceschi@iq.ufrgs.br; 00045960@ufrgs.br
Alzheimer's disease is known as a neurodegenerative disorder with major importance and the principal cause of dementia among the elderly. There is no cure for AD, but there are drugs that target the symptoms in order to improve the cognitive function of the patient. ${ }^{1,89}$

Four drugs are currently available for $\mathrm{AD}$ treatment and all were approved more than a decade ago. Of these, the first-line agents are the ChEIs (donepezil, rivastigmine, and galantamine). ${ }^{1}$ Tacrine (1,2,3,4-tetrahydroacridine, THA), was the first ChEI approved by the U.S. Food and Drug Administration (FDA) in 1993 for the palliative treatment of AD. ${ }^{5}$ However, it soon exhibited hepatotoxicity and consequently was withdrawn from the market shortly after its approval. ${ }^{10}$ 
Although the cause of AD is still not fully understood, dysregulation of the amyloid-beta $(A \beta)$ protein level and neurofibrillary tangles appear to be the predominant contributing factors. Paradoxically, previous clinical failures of anti-A $\beta$ antigens and $\gamma$-secretase inhibitors, and the recent clinical failures of $\beta$-secretase (BACE1) inhibitors and monoclonal anti-A $\beta$ antibodies, have led researchers to suggest dropping such therapies proposals. ${ }^{11}$

Although the role of $\mathrm{AChE}$ in cholinergic transmission is well known, the role of $\mathrm{BuChE}$ has not been sufficiently elucidated and an increasing number of studies ${ }^{3}$ have provided evidence suggesting that BuChE may play a distinct role in AD patients. Depending on the AD stage, there is a decline in $\mathrm{AChE}$ levels in the brain and a progressive increase of $\mathrm{BuChE}$ levels, which becomes responsible for acetylcholine hydrolysis. In the $\mathrm{AD}$ patients' brain, AChE levels are decreased by around 50\%, whereas BuChE levels increase by as much as $900 \% .^{12,13}$ $\mathrm{BuChE}$ is associated with insulin resistance, a typical feature of patients with type II diabetes. ${ }^{14}$ According to a recent mouse study, ${ }^{15} \mathrm{BuChE}$ regulates ghrelin levels to control social behavior, such as aggression. Thus, specific BuChE inhibitors may be considered as a new and promising therapeutic strategy for neurodegenerative diseases and others related to the cholinergic system. ${ }^{13,15}$

Different structural features of $\mathrm{AChE}$ and $\mathrm{BuChE}$ are related to their substrate specificity: $\mathrm{AChE}$ has higher selectivity for small molecules like $\mathrm{ACh}$, while $\mathrm{BuChE}$ is less substrate-specific, accommodating the metabolism of several different molecules, including various neuroactive peptides. ${ }^{16}$ Accordingly, the structural characteristic of BuChE provides a reasonable thought to design selective $\mathrm{BuChE}$ inhibitors. ${ }^{1,17}$ Furthermore, BuChE is associated with other pathological manifestations of $A D$, including the formation of $A \beta$ from the initially benign form seen in normal aging to malignant fibrillar A $\beta$ deposition. Thus, BuChE's inhibitors may serve the dual purpose of increasing acetylcholine levels and inhibiting fibrillar $\mathrm{A} \beta$ deposition. ${ }^{13,18-20}$

The active site gorge of $\mathrm{BuChE}$ is larger than that of AChE $(500 \AA \text { against } 300 \AA)^{3}$ and forms a bowl rather than a deep narrow gorge. ${ }^{21}$ Also, it has been shown that the gorge of BuChE contains about $40 \%$ fewer aromatic residues than $\mathrm{AChE}$, where they are substituted by smaller aliphatic or even polar residues. This accounts for most of the specific properties of BuChE. The acylation site, where catalytic reactions take place, is at the bottom of the gorge, about $20 \AA$ from the protein surface. It consists of the catalytic triad Ser198, His438, and Glu325. The triad of cholinesterase-related enzymes differs from that of serine proteases by having glutamate instead of aspartate. One of the most important features of the BuChE and $\mathrm{AChE}$ catalytic process is the $\mathrm{H}$-bond stabilization of the transition state by the oxyanion hole. The oxyanion hole is composed of three highly conserved $\mathrm{N}-\mathrm{H}$ dipoles from the main chain of residues Gly116, Gly117 and Ala119. ${ }^{22}$

The first designed hybrid molecule for the potential treatment of AD dates back to 1996 when Pang et al. ${ }^{23}$ reported the synthesis of alkylene-linked bis-tacrine compounds as a dual binding site inhibitor (DBS, namely compounds that are able to occupy the two binding sites). They envisaged that tacrine dimer targets the high affinity catalytic anionic site (CAS) and the low-affinity peripheral binding site (PAS) of AChE. These simple bis-tacrine dimer exhibited potency in orders 1,000 folds higher, compared to tacrine.

The literature reveals a plethora of studies of ChEIs based on DBS. A newly arising strategy is the synthesis of hybrids molecules, where two pharmacological molecules are mixed in one single molecule. These hybrids act as multi-target compounds, usually combining its potent cholinesterase inhibition with other pharmacological properties. ${ }^{24-29}$ However, the number of hybrids obtained from the combination of scaffolds different from those well-known ChEI drugs are quite restricted, and ChEIs with novel structural diversity are urgently needed.

The imidazolic nucleus is incorporated in many bioactive molecules playing a vital role in treating various types of diseases. ${ }^{30,31}$ The imidazole derivative 2,4,5-triphenyl-1H-imidazole, also known as lophine, can be used as fluorescent labeling reagents for amines, phenols, and carboxylic acids. ${ }^{32}$ The synthesis of various imidazoles and their derivatives are important targets in current years, among them 2,4,5-tri- and 1,2,4,5-tetrasubstituted imidazoles have received much attention. ${ }^{33-35}$ Also, hybrids containing lophine and pyrimidine nuclei connected by a methylene chain showed photophysical features that were successfully used to explore their interaction with bovine serum albumin (BSA) protein and exhibited significant suppression mechanism. ${ }^{36}$ In previous work, ${ }^{37}$ our group synthesized a series of bis $(n)$-lophine, where two lophine core were linked by a methylene chain. In this series, the bis(8)-lophine was the only active compound, showing a potent inhibition against $\mathrm{AChE}$ (half maximal inhibitory concentration $\left.\left(\mathrm{IC}_{50}\right)=42.55 \mathrm{nM}\right)$.

Among several methods reported in the literature for imidazoles synthesis, multicomponent reactions (MCRs) have received considerable attention and proves to be one of the most efficient methods for obtaining substituted imidazoles. In an MCR, a product is assembled according to a cascade of elementary chemical reactions. Thus, there is a network of reaction equilibria, which finally flow into an irreversible step yielding the product. The formation of a 
particular product is dependent on the reaction conditions: solvent, temperature, catalyst, concentration, the type of starting materials, and functional groups.

As part of our search for new ChEIs drug candidates, ${ }^{37-40}$ we report herein a new one-pot synthesis of bis $(n)$-lophine analogues as selective dual binding inhibitors of $\mathrm{BuChE}$ along with their cytotoxicity evaluation and molecular modeling studies.

\section{Experimental}

\section{Materials}

Microwave-assisted reactions were carried out using a MARS6 microwave oven (CEM Company, Charlotte, NC, USA) and the MARSXPress vessels with $10 \mathrm{~mL}$ of capacity. The temperature program used consisted in a heating ramp of $15 \mathrm{~min}$ to reach $110{ }^{\circ} \mathrm{C}$, followed by $1 \mathrm{~h}$ of reaction, with magnetic stirring. All melting points were determined in open glass capillaries using a Büchi M-565 (Essen, Germany) apparatus. Infrared (IR) spectra were recorded on a Varian 640-IR (Palo Alto, USA) spectrometer in $\mathrm{KBr}$ disks. ${ }^{1} \mathrm{H}$ nuclear magnetic resonance (NMR) and ${ }^{13} \mathrm{C}$ NMR spectra were recorded in $\mathrm{CDCl}_{3}$ solution on a Bruker BioSpin $400 \mathrm{MHz}$ (Billerica, USA) spectrometer. The relaxation time in both analyses was $1.0 \mathrm{~s}$ and the chemical shifts $(\delta)$ are given in part per million from the peak of tetramethylsilane ( $\delta \quad 0.00 \mathrm{ppm})$ as internal standard in ${ }^{1} \mathrm{H} \mathrm{NMR}$ or from the solvent peak of $\mathrm{CDCl}_{3}(\delta 77.16 \mathrm{ppm})$ in ${ }^{13} \mathrm{C}$ NMR APT (attached proton test). Multiplicities are given as s (singlet), d (doublet), dd (doublet of doublets), dt (doublet of triplets), t (triplet), td (triplet of doublets), m (multiplet); coupling constants $(J)$ are given in $\mathrm{Hz}$. High resolution mass spectrometry with electrospray ionization (HRMS-ESI) data on the positive mode was collected on a Micromass Q-Tof instrument from Waters (Manchester, UK). Samples were infused from a $100 \mathrm{~mL}$ Hamilton syringe at flow rate range from 5 to $10 \mathrm{~mL} \mathrm{~min}^{-1}$, depending on the sample. The instrument settings were the following: capillary voltage $3000 \mathrm{~V}$, cone voltage $33 \mathrm{~V}$, extraction cone voltage $2.5 \mathrm{~V}$, desolvation gas temperature $100{ }^{\circ} \mathrm{C}$. Nitrogen was used as the desolvation gas. Methanol (high performance liquid chromatography (HPLC) grade, Tedia, Fairfield, OH, USA) was used as solvent for the analyzed samples and filtered prior to injection. Purification by column chromatography was carried out on silica gel $60 \AA$ (70-230 mesh, Sigma-Aldrich, St. Louis, USA). Analytical thin layer chromatography (TLC) was conducted on aluminum plates with $0.2 \mathrm{~mm}$ of silica gel 60F-254 (Merck, Darmstadt, Germany). Dimethyl sulfoxide (DMSO), 5',5'-dithiobis-2-nitrobenzoic acid
(DTNB), acetylthiocholine iodide, heparin, dipotassium phosphate dibasic $\left(\mathrm{K}_{2} \mathrm{HPO}_{4}\right)$ and potassium phosphate monobasic $\left(\mathrm{KH}_{2} \mathrm{PO}_{4}\right)$ were obtained from Sigma-Aldrich (St. Louis, MO, USA). Solvents were obtained from Tedia (Fairfield, OH, USA) and Nuclear (Diadema, SP, Brazil), and reagents were purchased from Sigma-Aldrich, Acros Organics (Thermo Fisher Scientific, Waltham, Massachusetts, USA) and TCI (Tokyo, Japan).

\section{Synthesis}

General procedure for the synthesis of bis $(n)$-lophine homodimers (5-7)

A mixture of $1, n$-alkanediamine $(0.45 \mathrm{mmol})$, ammonium acetate $(0.90 \mathrm{mmol})$, benzil $(0.90 \mathrm{mmol})$ and $n$-pyridinecarboxaldehyde $(0.90 \mathrm{mmol})$ in absolute ethanol $(3.5 \mathrm{~mL})$ was added to a vial compatible with the use on the microwave reactor and the temperature program was started. This program was repeated three times in order to complete $4 \mathrm{~h}$ of reaction. At the end of the reaction time, the solvent was removed under reduced pressure. The crude product was purified by column chromatography (eluting with hexane:ethyl acetate, 90:10 with gradient elution until $0: 100)$ to give the desired product as a solid.

\section{1,6-Bis(4,5-diphenyl-2-(pyridin-2-yl)-1H-imidazol-1-yl) hexane (5a)}

Yellow solid; $39 \%$ yield; mp $75-76{ }^{\circ} \mathrm{C}$; IR (KBr) $v_{\max } / \mathrm{cm}^{-1}$ 3052, 2935, 2849, 1582, 1458, 788, 703; ${ }^{1} \mathrm{H}$ NMR (400 MHz, $\left.\mathrm{CDCl}_{3}\right) \delta 8.55-8.50(\mathrm{~m}, 2 \mathrm{H}), 8.41$ (d, $J 8.0 \mathrm{~Hz}, 2 \mathrm{H}), 7.81(\mathrm{td}, J 8.0,1.8 \mathrm{~Hz}, 2 \mathrm{H}), 7.58-7.50(\mathrm{~m}$, $4 \mathrm{H}), 7.48-7.43(\mathrm{~m}, 6 \mathrm{H}), 7.39-7.33(\mathrm{~m}, 4 \mathrm{H}), 7.27-7.21(\mathrm{~m}$, $6 \mathrm{H}), 7.20-7.14(\mathrm{~m}, 2 \mathrm{H}), 4.36(\mathrm{t}, J 7.6 \mathrm{~Hz}, 4 \mathrm{H}), 1.50-1.35(\mathrm{~m}$, 4H), 0.98-0.87 (m, 4H); ${ }^{13} \mathrm{C}$ NMR APT (100 MHz, $\mathrm{CDCl}_{3}$ ) $\delta$ 150.7, 148.3, 144.0, 137.7, 136.6, 134.2, 131.6, 131.1, 131.0, 129.0, 128.8, 128.1, 126.8, 126.4, 123.6, 122.5, 45.2, 30.6, 25.6; HRMS-ESI $m / z$, calcd. for $[\mathrm{M}+\mathrm{H}]^{+}: 677.3387$, found: 677.3386 .

\section{1,6-Bis(4,5-diphenyl-2-(pyridin-3-yl)-1H-imidazol-1-yl) hexane (5b)}

White solid; $37 \%$ yield; mp 194-195 ${ }^{\circ} \mathrm{C}$; IR (KBr) $v_{\max } / \mathrm{cm}^{-1} 3043,2927,2857,1473,773,696 ;{ }^{1} \mathrm{H}$ NMR $\left(400 \mathrm{MHz}, \mathrm{CDCl}_{3}\right) \delta 8.91-8.85(\mathrm{~m}, 2 \mathrm{H}), 8.69(\mathrm{dd}, J$ 4.8, $1.7 \mathrm{~Hz}, 2 \mathrm{H}), 8.08$ (dt, J 7.9, $1.7 \mathrm{~Hz}, 2 \mathrm{H}), 7.54-7.49$ (m, 4H), 7.49-7.42 (m, 8H), 7.37-7.32 (m, 4H), 7.26-7.14 (m, 6H), 3.79 (t, J 7.6 Hz, 4H), 1.22-1.11 (m, 4H), 0.70-0.61 (m, 4H); ${ }^{13} \mathrm{C}$ NMR APT $\left(100 \mathrm{MHz}, \mathrm{CDCl}_{3}\right) \delta 149.8,149.3,144.3$, 138.4, 136.7, 134.0, 130.9, 130.8, 130.2, 129.2, 128.9, 128.2, 127.5, 126.7, 126.6, 123.6, 44.5, 30.1, 25.3; HRMS-ESI $m / z$, calcd. for $[\mathrm{M}+\mathrm{H}]^{+}: 677.3387$, found: 677.3381 . 
1,6-Bis(4,5-diphenyl-2-(pyridin-4-yl)-1H-imidazol-1-yl) hexane $(5 \mathbf{c})$

White solid; 33\% yield; mp 200-201 ${ }^{\circ} \mathrm{C}$; IR (KBr) $v_{\max } / \mathrm{cm}^{-1} 3043,2927,2857,1597,835,765,703 ;{ }^{1} \mathrm{H}$ NMR $\left(400 \mathrm{MHz}, \mathrm{CDCl}_{3}\right) \delta 8.76-8.69(\mathrm{~m}, 4 \mathrm{H}), 7.63-7.58(\mathrm{~m}, 4 \mathrm{H})$, 7.53-7.43 (m, 10H), 7.38-7.32 (m, 4H), 7.27-7.15 (m, 6H), 3.84 (t, J7.6 Hz, 4H), 1.24-1.12 (m, 4H), 0.74-0.64 (m, 4H); ${ }^{13} \mathrm{C}$ NMR APT $\left(100 \mathrm{MHz}, \mathrm{CDCl}_{3}\right) \delta 150.3,144.5,138.8$, $133.9,131.0,130.8,130.7,129.2,129.0,128.1,126.7$, 126.7, 122.8, 44.6, 30.1, 25.3; HRMS-ESI $\mathrm{m} / \mathrm{z}$, calcd. for $[\mathrm{M}+\mathrm{H}]^{+}:$677.3387, found: 677.3382 .

1,7-Bis(4,5-diphenyl-2-(pyridin-2-yl)-1H-imidazol-1-yl) heptane (6a)

Yellow solid; $45 \%$ yield; mp $149-150{ }^{\circ} \mathrm{C}$; IR (KBr) $v_{\max } / \mathrm{cm}^{-1} 3055,2946,2860,1586,1469,693 ;{ }^{1} \mathrm{H}$ NMR $\left(400 \mathrm{MHz}, \mathrm{CDCl}_{3}\right) \delta 8.55-8.49(\mathrm{~m}, 2 \mathrm{H}), 8.35-8.29(\mathrm{~m}, 2 \mathrm{H})$, $7.76(\mathrm{td}, J 7.8,1.8 \mathrm{~Hz}, 2 \mathrm{H}), 7.53-7.47(\mathrm{~m}, 4 \mathrm{H}), 7.46-7.32$ $(\mathrm{m}, 11 \mathrm{H}), 7.23-7.16(\mathrm{~m}, 6 \mathrm{H}), 7.16-7.11(\mathrm{~m}, 2 \mathrm{H}), 4.37$ (t, $J 7.6 \mathrm{~Hz}, 4 \mathrm{H}), 1.51-1.35(\mathrm{~m}, 4 \mathrm{H}), 1.00-0.81(\mathrm{~m}, 6 \mathrm{H})$; ${ }^{13} \mathrm{C}$ NMR APT $\left(75 \mathrm{MHz}, \mathrm{CDCl}_{3}\right) \delta 151.1,148.2,144.1$, 137.9, 136.5, 134.6, 131.7, 131.3, 131.2, 128.9, 128.7, 128.1, 126.7, 126.3, 123.4, 122.4, 45.2, 30.7, 28.0, 26.1; HRMS-ESI $\mathrm{m} / \mathrm{z}$, calcd. for $[\mathrm{M}+\mathrm{H}]^{+}: 691.3544$, found: 695.3543.

1,7-Bis(4,5-diphenyl-2-(pyridin-3-yl)-1H-imidazol-1-yl) heptane (6b)

Yellow solid; $39 \%$ yield; mp $76-77{ }^{\circ} \mathrm{C}$; IR $(\mathrm{KBr})$ $v_{\max } / \mathrm{cm}^{-1} 3070,2931,2853,1484,1026,770 ;{ }^{1} \mathrm{H}$ NMR $\left(400 \mathrm{MHz}, \mathrm{CDCl}_{3}\right) \delta 8.97-8.84(\mathrm{~m}, 2 \mathrm{H}), 8.72-8.60(\mathrm{~m}, 2 \mathrm{H})$, 8.04 (dt, J 7.9, $1.8 \mathrm{~Hz}, 2 \mathrm{H}), 7.55-7.31(\mathrm{~m}, 16 \mathrm{H}), 7.24-7.11$ (m, 6H), 3.80 (t, J 7.6 Hz, 4H), 1.27-1.14 (m, 4H), 0.77-0.59 $(\mathrm{m}, 6 \mathrm{H}) ;{ }^{13} \mathrm{C}$ NMR APT $\left(100 \mathrm{MHz}, \mathrm{CDCl}_{3}\right) \delta 149.9,149.5$, 144.5, 138.8, 136.8, 134.2, 131.2 (6), 131.2 (1), 130.4, 129.3, 129.0, 128.3, 127.9, 126.9, 126.8, 123.7, 44.8, 30.5, 27.8, 26.0; HRMS-ESI $\mathrm{m} / z$, calcd. for [M+ H] $]^{+}$691.3544, found: 691.3543 .

1,7-Bis(4,5-diphenyl-2-(pyridin-4-yl)-1H-imidazol-1-yl) heptane $(6 \mathbf{c})$

White solid; $31 \%$ yield; mp $164-165{ }^{\circ} \mathrm{C}$; IR (KBr) $v_{\max } / \mathrm{cm}^{-1} 3055,2922,2853,1601,1415,972 ;{ }^{1} \mathrm{H}$ NMR $\left(400 \mathrm{MHz}, \mathrm{CDCl}_{3}\right) \delta$ 8.76-8.67 (m, 1H), 7.65-7.60 (m, $1 \mathrm{H}), 7.51-7.41(\mathrm{~m}, 3 \mathrm{H}), 7.39-7.32(\mathrm{~m}, 1 \mathrm{H}), 7.24-7.12(\mathrm{~m}$, 2H), $3.88(\mathrm{t}, J 7.6 \mathrm{~Hz}, 1 \mathrm{H}), 1.29-1.18(\mathrm{~m}, 1 \mathrm{H}), 0.81-0.63$ $(\mathrm{m}, 1 \mathrm{H}) ;{ }^{13} \mathrm{C}$ NMR APT $\left(75 \mathrm{MHz}, \mathrm{CDCl}_{3}\right) \delta 150.4,144.6$, 139.0, 134.1, 131.2, 131.1, 131.0, 129.4, 129.2, 128.3, 126.9, 126.8, 44.9, 30.4, 27.9, 26.0; HRMS-ESI $\mathrm{m} / \mathrm{z}$, calcd. for $[\mathrm{M}+\mathrm{H}]^{+}:$691.3544, found: 691.3544 .
1,8-Bis(4,5-diphenyl-2-(pyridin-2-yl)-1H-imidazol-1-yl) octane (7a)

Yellow solid; 55\% yield; mp $147-148{ }^{\circ} \mathrm{C}$; IR (KBr) $v_{\max } / \mathrm{cm}^{-1} 3031,2931,2845,1578,1477,700 ;{ }^{1} \mathrm{H}$ NMR $\left(400 \mathrm{MHz}, \mathrm{CDCl}_{3}\right) \delta 8.57-8.51(\mathrm{~m}, 2 \mathrm{H}), 8.35(\mathrm{~d}, J 7.8 \mathrm{~Hz}$, $2 \mathrm{H}), 7.77(\mathrm{td}, J 7.8,1.8 \mathrm{~Hz}, 2 \mathrm{H}), 7.54-7.49(\mathrm{~m}, 4 \mathrm{H})$, 7.46-7.40 (m, 6H), 7.39-7.34 (m, 4H), 7.23-7.17 (m, 6H), 7.17-7.10 (m, 2H), $4.39(\mathrm{t}, J 7.6 \mathrm{~Hz}, 4 \mathrm{H}), 1.59-1.41(\mathrm{~m}$, $4 \mathrm{H}), 1.09-0.74(\mathrm{~m}, 8 \mathrm{H}) ;{ }^{13} \mathrm{C}$ NMR APT $\left(75 \mathrm{MHz}, \mathrm{CDCl}_{3}\right)$ $\delta$ 151.2, 148.4, 144.3, 138.0, 136.7, 134.8, 131.9, 131.4(5), 131.4 (2), 129.0, 128.3, 126.7, 123.6, 122.6, 45.4, 31.0, 28.6, 26.4; HRMS-ESI $\mathrm{m} / \mathrm{z}$, calcd. for $[\mathrm{M}+\mathrm{H}]^{+}:$705.3700, found: 705.3701 .

1,8-Bis(4,5-diphenyl-2-(pyridin-3-yl)-1H-imidazol-1-yl) octane $(7 \mathbf{b})$

Yellow solid; $47 \%$ yield; mp $170-171{ }^{\circ} \mathrm{C}$; IR (KBr) $v_{\max } / \mathrm{cm}^{-1} 3055,2931,2860,1500,1314,715 ;{ }^{1} \mathrm{H}$ NMR $\left(400 \mathrm{MHz}, \mathrm{CDCl}_{3}\right) \delta$ 8.94-8.87 (m, 2H), 8.65 (dd, $J 4.8$, $1.6 \mathrm{~Hz}, 2 \mathrm{H}), 8.04(\mathrm{dt}, J 7.6,1.6 \mathrm{~Hz}, 2 \mathrm{H}), 7.52-7.47(\mathrm{~m}, 5 \mathrm{H})$, 7.45-7.34 (m, 11H), 7.24-7.11 (m, 6H), $3.83(\mathrm{t}, J 7.6 \mathrm{~Hz}$, $4 \mathrm{H}), 1.38-1.13(\mathrm{~m}, 4 \mathrm{H}), 0.96-0.65(\mathrm{~m}, 8 \mathrm{H}) ;{ }^{13} \mathrm{C}$ NMR APT $\left(100 \mathrm{MHz}, \mathrm{CDCl}_{3}\right) \delta 149.9,149.6,144.5,138.6,136.8$, 134.4, 131.2, 131.1, 130.4, 129.3, 129.0, 128.3, 127.9, 126.9, 126.7, 123.7, 44.9, 30.5, 28.4, 26.10; HRMS-ESI $\mathrm{m} / z$, calcd. for $[\mathrm{M}+\mathrm{H}]^{+}:$705.3700, found: 705.3709.

1,8-Bis(4,5-diphenyl-2-(pyridin-4-yl)-1H-imidazol-1-yl) octane (7c)

White solid; $35 \%$ yield; mp $170-171{ }^{\circ} \mathrm{C}$; IR (KBr) $v_{\max } / \mathrm{cm}^{-1} 3032,2931,2854,1597,1419,964,702 ;{ }^{1} \mathrm{H}$ NMR $\left(400 \mathrm{MHz}, \mathrm{CDCl}_{3}\right) \delta 8.73-8.68(\mathrm{~m}, 4 \mathrm{H}), 7.66-7.62(\mathrm{~m}, 4 \mathrm{H})$, 7.52-7.42 (m, 14H), 7.39-7.34 (m, 6H), $3.91(\mathrm{t}, J 7.6 \mathrm{~Hz}$, $4 \mathrm{H}), 1.34-1.24(\mathrm{~m}, 4 \mathrm{H}), 0.87-0.72(\mathrm{~m}, 8 \mathrm{H}) ;{ }^{13} \mathrm{C}$ NMR APT $\left(100 \mathrm{MHz}, \mathrm{CDCl}_{3}\right) \delta 150.1,144.4,138.9,138.8,134.0$, 131.1, 130.9, 130.8, 129.1, 129.0, 128.1, 126.8, 126.6, 122.9, 44.8, 30.3, 28.2, 25.9; HRMS-ESI $\mathrm{m} / \mathrm{z}$, calcd. for $[\mathrm{M}+\mathrm{H}]^{+}:$705.3700, found: 705.3702 .

\section{General procedure for the synthesis of $N$-alkylaminolophine (9a-9c)}

A mixture of $1, n$-alkanediamine ( $1 \mathrm{mmol})$, ammonium acetate $(1 \mathrm{mmol})$, benzil $(1 \mathrm{mmol})$ and benzaldehyde $(1 \mathrm{mmol})$ in absolute ethanol $(3.5 \mathrm{~mL})$ was added to a vial compatible with the use on the microwave reactor and the temperature program was started. This program was repeated three times in order to complete $4 \mathrm{~h}$ of reaction. At the end of the reaction time, the solvent was removed under reduced pressure. The resultant oil was dissolved in dichloromethane $(20 \mathrm{~mL})$ and washed with a mixture of water and a saturated solution of $\mathrm{NaCl} 1: 1(2 \times 20 \mathrm{~mL})$. 
The organic phase was dried with anhydrous $\mathrm{Na}_{2} \mathrm{SO}_{4}$ and the solvent was removed under reduced pressure. The crude product was purified by column chromatography (eluting with chloroform-methanol-ammonium hydroxide, 98:1.5:0.5 with gradient elution until 80:19.5:0.5) to give the desired product as a yellow oil (47\% yield).

\section{General procedure for the synthesis of lophine heterodimers (10-12)}

A mixture of $N$-alkylaminolophine $(0.45 \mathrm{mmol})$, ammonium acetate $(0.45 \mathrm{mmol})$, benzil $(0.45 \mathrm{mmol})$ and $n$-pyridinecarboxaldehyde $(0.45 \mathrm{mmol})$ in absolute ethanol $(3.5 \mathrm{~mL})$ was added to a vial compatible with the use on the microwave reactor and the temperature program was started. This program was repeated three times in order to complete $4 \mathrm{~h}$ of reaction. At the end of the reaction time, the solvent was removed under reduced pressure. The crude product was purified by column chromatography (eluting with hexane:ethyl acetate, 90:10 with gradient elution until $0: 100$ ) to give the desired product as solid.

2-(4,5-Diphenyl-1-(6-(2,4,5-triphenyl-1H-imidazol-1-yl) hexyl)-1H-imidazol-2-yl)pyridine (10a)

Yellow solid; $37 \%$ yield; mp 78-79 ${ }^{\circ} \mathrm{C}$; IR (KBr) $v_{\max } / \mathrm{cm}^{-1}$ 3059, 2926, 2857, 1586, 1464, 771, 691; ${ }^{1} \mathrm{H}$ NMR $\left(400 \mathrm{MHz}, \mathrm{CDCl}_{3}\right) \delta 8.52-8.49(\mathrm{~m}, 1 \mathrm{H}), 8.35-8.31$ (m, 1H), $7.77(\mathrm{td}, J 7.6,2.0 \mathrm{~Hz}, 1 \mathrm{H}), 7.66-7.61(\mathrm{~m}, 2 \mathrm{H})$, 7.54-7.48 (m, 4H), 7.47-7.39 (m, 9H), 7.37-7.30 (m, 4H), $7.23-7.10(\mathrm{~m}, 7 \mathrm{H}), 4.29(\mathrm{t}, J 7.6 \mathrm{~Hz}, 2 \mathrm{H}), 3.78(\mathrm{t}, J 7.6 \mathrm{~Hz}$, $2 \mathrm{H}), 1.38-1.27$ (m, 2H), 1.24-1.13 (m, 2H), 0.81-0.70 (m, $4 \mathrm{H}) ;{ }^{13} \mathrm{C}$ NMR APT $\left(100 \mathrm{MHz}, \mathrm{CDCl}_{3}\right) \delta$ 151.0, 148.2, $147.6,144.0,137.9,137.7,136.6,134.5,131.6,131.5$, $131.4,131.2,131.1,130.9,129.8,129.5,129.1,129.0$, $128.9,128.8,128.7,128.6,128.5,128.1,128.0,126.8$, $126.7,126.3,126.2,123.4,122.4,45.0,44.5,30.5,30.1$, 25.5, 25.4; HRMS-ESI $m / z$, calcd. for $[\mathrm{M}+\mathrm{H}]^{+}: 676.3435$, found: 676.3416 .

3-(4,5-Diphenyl-1-(6-(2,4,5-triphenyl-1H-imidazol-1-yl) hexyl)-1H-imidazol-2-yl)pyridine (10b)

Yellow solid; $25 \%$ yield; mp $167-168{ }^{\circ} \mathrm{C}$; IR (KBr) $v_{\max } / \mathrm{cm}^{-1} 3059,2927,2857,1480,773,687 ;{ }^{1} \mathrm{H}$ NMR $\left(400 \mathrm{MHz}, \mathrm{CDCl}_{3}\right) \delta 8.92-8.86(\mathrm{~m}, 1 \mathrm{H}), 8.68(\mathrm{dd}, J$ 4.8, $1.6 \mathrm{~Hz}, 1 \mathrm{H}), 8.05-8.00(\mathrm{~m}, 1 \mathrm{H}), 7.65-7.61(\mathrm{~m}, 2 \mathrm{H})$, 7.54-7.49 (m, 4H), 7.48-7.40 (m, 10H), 7.38-7.32 (m, 4H), 7.26-7.12 (m, 6H), 3.82-3.71 (m, 4H), 1.19-1.06 (m, 4H), 0.71-0.55 (m, 4H); ${ }^{13} \mathrm{C}$ NMR APT (100 MHz, $\left.\mathrm{CDCl}_{3}\right) \delta$ $149.7,149.3,147.6,144.3,138.5,137.7,136.6,134.4$, $134.1,131.4,131.3,131.0,130.9,130.8,130.2,129.5$, 129.1, 129.1, 129.0, 128.9, 128.8, 128.6, 128.5, 128.1,
128.0, 127.7, 126.8, 126.7, 126.5, 126.3, 123.5, 44.6, 44.3, $30.1,29.9,25.3,25.3$; HRMS-ESI $m / z$, calcd. for $[\mathrm{M}+\mathrm{H}]^{+}$: 676.3435 , found: 676.3424 .

4-(4,5-Diphenyl-1-(6-(2,4,5-triphenyl-1H-imidazol-1-yl) hexyl)-1H-imidazol-2-yl)pyridine (10c)

Yellow solid; $28 \%$ yield; mp 202-203 ${ }^{\circ} \mathrm{C}$; IR (KBr) $v_{\max } / \mathrm{cm}^{-1} 3052,2927,2849,1597,773,703 ;{ }^{1} \mathrm{H} \mathrm{NMR}$ $\left(400 \mathrm{MHz}, \mathrm{CDCl}_{3}\right) \delta 8.71-8.68(\mathrm{~m}, 2 \mathrm{H}), 7.63-7.59(\mathrm{~m}$, $2 \mathrm{H}), 7.59-7.57(\mathrm{~m}, 2 \mathrm{H}), 7.52-7.39(\mathrm{~m}, 13 \mathrm{H}), 7.36-7.29(\mathrm{~m}$, $4 \mathrm{H}), 7.24-7.10(\mathrm{~m}, 6 \mathrm{H}), 3.83-3.72(\mathrm{~m}, 4 \mathrm{H}), 1.18-1.06(\mathrm{~m}$, 4H), 0.68-0.57 (m, 4H); $\left.{ }^{13} \mathrm{C} \mathrm{NMR} \mathrm{APT} \mathrm{(100} \mathrm{MHz,} \mathrm{CDCl}_{3}\right)$ $\delta$ 150.2, 147.6, 144.4, 138.8, 138.7, 137.7, 134.4, 134.0, $131.4,131.3,131.0,130.9,130.8,130.7,129.4,129.1(5)$, 129.1 (2), 129.0, 128.8, 128.6, 128.1, 128.0, 126.7, 126.6, 126.3, 122.8, 44.6, 44.3, 30.1, 29.9, 25.3, 25.3; HRMS-ESI $\mathrm{m} / \mathrm{z}$, calcd. for $[\mathrm{M}+\mathrm{H}]^{+}:$676.3435, found: 676.3420 .

2-(4,5-Diphenyl-1-(7-(2,4,5-triphenyl-1H-imidazol-1-yl) heptyl)-1H-imidazol-2-yl)pyridine (11a)

Yellow solid; $49 \%$ yield; mp $165-166{ }^{\circ} \mathrm{C}$; IR (KBr) $v_{\max } / \mathrm{cm}^{-1} 3052,2935,2849,1589,781,696 ;{ }^{1} \mathrm{H}$ NMR $\left(400 \mathrm{MHz}, \mathrm{CDCl}_{3}\right) \delta 8.55-8.52(\mathrm{~m}, 1 \mathrm{H}), 8.35(\mathrm{dt}, J$ 8.0, $1.0 \mathrm{~Hz}, 1 \mathrm{H}), 7.79(\mathrm{td}, J 7.6,2.0 \mathrm{~Hz}, 1 \mathrm{H}), 7.70-7.65(\mathrm{~m}, 2 \mathrm{H})$, 7.56-7.50 (m, 4H), 7.49-7.42 (m, 9H), 7.42-7.35 (m, 4H), 7.26-7.19 (m, 5H), 7.18-7.13 (m, 2H), $4.36(\mathrm{t}, J 7.6 \mathrm{~Hz}, 2 \mathrm{H})$, $3.83(\mathrm{t}, J 7.6 \mathrm{~Hz}, 2 \mathrm{H}), 1.45-1.35(\mathrm{~m}, 2 \mathrm{H}), 1.30-1.19$ (m, 2H), 0.91-0.83 (m, 2H), 0.81-0.72 (m, 4H); ${ }^{13} \mathrm{C}$ NMR APT $\left(100 \mathrm{MHz}, \mathrm{CDCl}_{3}\right) \delta 151.0,148.2,147.6,144.1,137.9$, $137.7,136.6,134.5,131.6,131.5,131.4,131.3,131.2$, $131.0,129.5,129.1,129.0,128.9,128.8,128.7,128.6$, $128.1,128.0,126.8,126.7,126.3,126.2,123.4,122.4,45.1$, 44.5, 30.6, 30.2, 27.8, 26.0, 25.9; HRMS-ESI m/z, calcd. for $[\mathrm{M}+\mathrm{H}]^{+}:$690.3591, found: 690.3586 .

3-(4,5-Diphenyl-1-(7-(2,4,5-triphenyl-1H-imidazol-1-yl) heptyl)-1H-imidazol-2-yl)pyridine (11b)

Yellow solid; $23 \%$ yield; mp 78-79 ${ }^{\circ} \mathrm{C}$; IR (KBr) $v_{\max } / \mathrm{cm}^{-1}$ 3432, 3053, 2939, 2843, 1491, 786, 700; ${ }^{1} \mathrm{H}$ NMR (400 MHz, $\left.\mathrm{CDCl}_{3}\right) \delta 8.92(\mathrm{~d}, J 1.8 \mathrm{~Hz}, 1 \mathrm{H})$, $8.68(\mathrm{dd}, J 4.8,1.8 \mathrm{~Hz}, 1 \mathrm{H}), 8.05$ (dt, J 7.9, $1.8 \mathrm{~Hz}, 1 \mathrm{H})$, 7.69-7.62 (m, 2H), 7.57-7.34 (m, 18H), 7.26-7.12 (m, 6H), $3.81(\mathrm{t}, J 7.6 \mathrm{~Hz}, 4 \mathrm{H}), 1.27-1.13(\mathrm{~m}, 4 \mathrm{H}), 0.82-0.61(\mathrm{~m}$, $6 \mathrm{H}) ;{ }^{13} \mathrm{C}$ NMR APT $\left(100 \mathrm{MHz}, \mathrm{CDCl}_{3}\right) \delta 149.7,149.4$, $147.6,144.3,138.5,137.7,136.6,134.5,134.1,131.5$, $131.4,131.0,130.9,130.8,130.2,129.5,129.2,129.1$, $129.0,128.9,128.8,128.6,128.1,128.0,127.7,126.8$, $126.7,126.5,126.2,123.5,44.7,44.4,30.3,30.0,27.6$, 25.8, 25.7; HRMS-ESI $m / z$, calcd. for $[\mathrm{M}+\mathrm{H}]^{+}:$690.3591, found: 690.3588 . 
4-(4,5-Diphenyl-1-(7-(2,4,5-triphenyl-1H-imidazol-1-yl) heptyl)-1H-imidazol-2-yl)pyridine (11c)

Yellow solid; $26 \%$ yield; $\mathrm{mp} 152-153{ }^{\circ} \mathrm{C}$; IR (KBr) $v_{\max } / \mathrm{cm}^{-1} 3043,2919,2857,1597,781,687 ;{ }^{1} \mathrm{H}$ NMR $\left(400 \mathrm{MHz}, \mathrm{CDCl}_{3}\right) \delta 8.75-8.71(\mathrm{~m}, 2 \mathrm{H}), 7.68-7.62(\mathrm{~m}$, $4 \mathrm{H}), 7.55-7.42(\mathrm{~m}, 13 \mathrm{H}), 7.41-7.34(\mathrm{~m}, 4 \mathrm{H}), 7.26-7.12$ (m, 6H), 3.92-3.79 (m, 4H), 1.31-1.14 (m, 4H), 0.80-0.61 $(\mathrm{m}, 6 \mathrm{H}) ;{ }^{13} \mathrm{C}$ NMR APT $\left(75 \mathrm{MHz}, \mathrm{CDCl}_{3}\right) \delta 150.2,147.6$, $144.4,138.9,138.8,137.7,134.5,134.0,131.5,131.0$, $130.9,130.9,130.8,129.5,129.2,129.1,129.0,129.0$, 128.8, 128.6, 128.1, 128.0, 126.7, 126.6, 126.2, 122.8, 44.7, 44.4, 30.2, 30.0, 27.7, 25.8, 25.7; HRMS-ESI $\mathrm{m} / \mathrm{z}$, calcd. for $[\mathrm{M}+\mathrm{H}]^{+}:$690.3591, found: 690.3592 .

\section{2-(4,5-Diphenyl-1-(8-(2,4,5-triphenyl-1H-imidazol-1-yl)} octyl)-1H-imidazol-2-yl)pyridine (12a)

Yellow solid; $35 \%$ yield; mp $115-116{ }^{\circ} \mathrm{C}$; IR (KBr) $v_{\max } / \mathrm{cm}^{-1} 3043,2927,2849,1589,1504,788,703$; ${ }^{1} \mathrm{H}$ NMR $\left(400 \mathrm{MHz}, \mathrm{CDCl}_{3}\right) \delta 8.58-8.54(\mathrm{~m}, 1 \mathrm{H}), 8.36(\mathrm{dt}$, $J$ 8.0, $1.0 \mathrm{~Hz}, 1 \mathrm{H}), 7.80(\mathrm{td}, J 7.6,2.0 \mathrm{~Hz}, 1 \mathrm{H}), 7.72-7.66$ $(\mathrm{m}, 2 \mathrm{H}), 7.57-7.51(\mathrm{~m}, 4 \mathrm{H}), 7.50-7.36(\mathrm{~m}, 13 \mathrm{H}), 7.26-7.19$ (m, 5H), 7.18-7.12 (m, 2H), $4.41(\mathrm{t}, J 7.6 \mathrm{~Hz}, 2 \mathrm{H}), 3.86(\mathrm{t}$, J 7.6 Hz, 2H), 1.51-1.40 (m, 2H), 1.34-1.21 (m, 3H), 0.990.89 (m, 2H), 0.87-0.74 (m, 6H); ${ }^{13} \mathrm{C}$ NMR APT $(100 \mathrm{MHz}$, $\left.\mathrm{CDCl}_{3}\right) \delta 151.1,148.2,147.6,144.1,137.9,137.7,136.5$, 134.6, 134.5, 131.7, 131.6, 131.5, 131.3, 131.2, 131.0, $129.6,129.1,129.0,128.9,128.8,128.7,128.6,128.1$, $128.0,126.8,126.7,126.3,126.2,123.5,122.4,45.2,44.6$, 30.7, 30.2, 28.3, 28.2, 26.1, 25.9; HRMS-ESI $\mathrm{m} / \mathrm{z}$, calcd. for $[\mathrm{M}+\mathrm{H}]^{+}:$704.3748, found: 704.3739.

3-(4,5-Diphenyl-1-(8-(2,4,5-triphenyl-1H-imidazol-1-yl) octyl)- $1 \mathrm{H}$-imidazol-2-yl)pyridine (12b)

Yellow solid; $31 \%$ yield; mp $140-141{ }^{\circ} \mathrm{C}$; IR (KBr) $v_{\max } / \mathrm{cm}^{-1} 3059,2926,2852,1475,760,696 ;{ }^{1} \mathrm{H}$ NMR $\left(400 \mathrm{MHz}, \mathrm{CDCl}_{3}\right) \delta$ 8.95-8.93 (m, 1H), 8.68 (dd, $J$ 4.8, $1.8 \mathrm{~Hz}, 1 \mathrm{H}), 8.07(\mathrm{dt}, J 8.0,1.8 \mathrm{~Hz}, 1 \mathrm{H}), 7.70-7.66(\mathrm{~m}, 2 \mathrm{H})$, 7.55-7.51 (m, 4H), 7.50-7.38 (m, 14H), 7.26-7.12 (m, 6H), 3.89-3.81 (m, 4H), 1.32-1.19 (m, 5H), 0.86-0.68 (m, 9H); ${ }^{13} \mathrm{C}$ NMR APT $\left(100 \mathrm{MHz}, \mathrm{CDCl}_{3}\right) \delta 149.7,149.4,147.6$, 144.3, 138.5, 137.7, 136.6, 134.5, 134.2, 131.6, 131.5, 131.1, $131.0,130.9,130.3,129.5,129.2,129.1,129.0,128.9,128.8$, $128.6,128.1,128.0,127.7,126.8,126.7,126.5,126.2,123.5$, 44.7, 44.5, 30.4, 30.1, 28.2, 28.1, 25.9, 25.9; HRMS-ESI $\mathrm{m} / \mathrm{z}$, calcd. for $[\mathrm{M}+\mathrm{H}]^{+}: 704.3748$ found 704.3729 .

4-(4,5-Diphenyl-1-(8-(2,4,5-triphenyl-1H-imidazol-1-yl) octyl)-1H-imidazol-2-yl)pyridine (12c)

Yellow solid; $47 \%$ yield; mp $140-141{ }^{\circ} \mathrm{C}$; IR (KBr) $v_{\max } / \mathrm{cm}^{-1} 3043,2927,2842,1597,773,703 ;{ }^{1} \mathrm{H}$ NMR $\left(400 \mathrm{MHz}, \mathrm{CDCl}_{3}\right) \delta 8.76-8.71(\mathrm{~m}, 2 \mathrm{H}), 7.71-7.64(\mathrm{~m}$,
4H), 7.56-7.37 (m, 17H), 7.26-7.12 (m, 6H), 3.95-3.82 (m, $4 \mathrm{H}), 1.36-1.19$ (m, 4H), 0.89-0.68 (m, 8H); ${ }^{13} \mathrm{C}$ NMR APT $\left(100 \mathrm{MHz}, \mathrm{CDCl}_{3}\right) \delta 150.2,147.5,144.4,138.9,138.7$, $137.5,134.3,134.0,131.4,131.2,131.1,131.0,130.9$, $130.8,129.5,129.2,129.1,129.0,129.0,128.9,128.7$, 128.6, 128.1, 128.0, 126.9, 126.8, 126.6, 126.3, 122.9, 44.8, 44.6, 30.3, 30.1, 28.2, 28.1, 25.9, 25.9; HRMS-ESI $\mathrm{m} / \mathrm{z}$, calcd. for $[\mathrm{M}+\mathrm{H}]^{+}$: 704.3748, found: 704.3747.

\section{Cholinesterase inhibition assay}

Electric eel $\mathrm{AChE}$ and horse serum BuChE were used as sources of both cholinesterases. AChE and BuChE inhibitory activities were measured in vitro by the spectrophotometric method developed by Ellman, ${ }^{41}$ with slight modifications. The lyophilized enzymes, $500 \mathrm{U}$ AChE and $300 \mathrm{U}$ BuChE, were dissolved in buffer phosphate A $\left(8 \mathrm{mM} \mathrm{K}_{2} \mathrm{HPO}_{4}, 2.3 \mathrm{mM} \mathrm{NaH}_{2} \mathrm{PO}_{4}\right)$ to obtain 5 and $3 \mathrm{U} \mathrm{mL}^{-1}$ stock solution, respectively. Further enzymes dilution were carried out with buffer phosphate $\mathrm{B}$ (8 $\mathrm{mM} \mathrm{K}_{2} \mathrm{HPO}_{4}, 2.3 \mathrm{mM} \mathrm{NaH}_{2} \mathrm{PO}_{4}, 0.15 \mathrm{M} \mathrm{NaCl}, 0.05 \%$ Tween 20, pH 7.6) to produce 0.5 and $0.3 \mathrm{U} \mathrm{mL}^{-1}$ enzyme solution, respectively. Samples were dissolved in buffer phosphate B with $1.25 \% \mathrm{CHCl}_{3}$ and $13.75 \%$ of $\mathrm{MeOH}$ as a cosolvent mixture. $300 \mu \mathrm{L}$ of enzyme solution and $300 \mu \mathrm{L}$ of sample solution were mixed in a test tube and incubated for 60 or $120 \mathrm{~min}$ at room temperature. The reaction was started by adding $600 \mu \mathrm{L}$ of the substrate solution ( $0.5 \mathrm{mM}$ DTNB, $0.6 \mathrm{mM}$ acetylthiocholine iodide/ butyrylthiocholine iodide (ATCI/BTCI), $0.1 \mathrm{M} \mathrm{Na}_{2} \mathrm{HPO}_{4}$, $\mathrm{pH} 7.5$ ), and the absorbance was read at $405 \mathrm{~nm}$ for $120 \mathrm{~s}$ at $25{ }^{\circ} \mathrm{C}$. Enzymes activity was calculated by comparing reaction rates for the samples to the blank. All reactions were performed in triplicate, and the $\mathrm{IC}_{50}$ values were determined with GraphPad Prism 5. ${ }^{42}$ Tacrine (99\%) was used as the reference inhibitor for $\mathrm{AChE}$ and BuChE.

\section{Kinetic characterization of BuChE inhibition}

The enzyme reaction was carried out at three fixed inhibitor (11b) concentrations (0, 0.15 and $0.055 \mu \mathrm{M})$. In each case, the initial velocity measurements were obtained at varying substrate (S) concentrations ([BTCI] $15-450 \mu \mathrm{M})$, and the reciprocal of the initial velocity $(1 / v)$ was plotted as a function of $1 /[\mathrm{S}]$. The data were analyzed with GraphPad Prism 5. ${ }^{42}$ The Lineweaver-Burk plot showed a pattern of lines with increasing slopes, characteristic of non-competitive type inhibition (inhibition constant (Ki) $21.29 \mathrm{nM}$ ). The nonlinear regression of these data fitted with non-competitive inhibition with a coefficient of determination $\mathrm{R}^{2}=0.9830$. 


\section{Evaluation of cytotoxicity in in vitro models}

The Vero (African green monkey kidney cells), HepG2 (liver hepatocellular carcinoma), and C6 (astroglial) cell lines were purchased from BCRJ (Rio de Janeiro Cell Bank). All cells were maintained in Dulbecco's modified Eagle medium (DMEM) supplemented with 10\% fetal bovine serum (FBS, Vero and HepG2 cells) or 5\% FBS (C6 cells), $0.5 \%$ amphotericin B $250 \mu \mathrm{g} \mathrm{mL}^{-1}, 0.5 \%$ penicillin $100 \mathrm{IU} \mathrm{mL}^{-1}$ and streptomycin $10 \mathrm{mg} \mathrm{mL}^{-1}$, at $37{ }^{\circ} \mathrm{C}$, in a humid atmosphere containing $5 \%$ of $\mathrm{CO}_{2}$. The cells were seeded in 96-well plates $\left(3 \times 10^{4}\right.$ cell well $^{-1}$ for $\mathrm{C} 6$, and $2 \times 10^{4}$ cell well $^{-1}$ for Vero and HepG2) and incubated for $24 \mathrm{~h}$. For cytotoxic studies, the selected compounds were dissolved in culture medium containing $0.5 \%$ DMSO, and the cells were exposed to them in concentrations up to $125 \mu \mathrm{M}$ (compounds $\mathbf{5 b}$ and $\mathbf{1 2 b}$ ) or $500 \mu \mathrm{M}$ (tacrine), for $24 \mathrm{~h}$. The cell viability was determined by the MTT (3-(4,5-dimethylthiazol-2-yl)-2,5-diphenyltetrazolium bromide) assay. ${ }^{43}$ At least three independent experiments were performed in triplicate for each trial $(n=3)$. The results were expressed as $\mathrm{IC}_{50}$ (50\% inhibiting concentration) compared to the control (vehicle DMSO at $0.5 \%$ ). The GraphPad Prism $5^{42}$ program was used for statistical analysis.

\section{Molecular docking}

The three-dimensional structures of the compounds were prepared using the Maestro Suite ${ }^{44}$ and isomers, protonation states, and tautomers of the ligands were determined using LigPrep/Epik from Maestro at $\mathrm{pH} 7.4 \pm 0.4 .^{45,46}$ The AChE and BuChE structures were prepared with the Protein Preparation Wizard tool from Maestro. ${ }^{47}$ The protonation states of the amino acid residues were determined using PROPKA at $\mathrm{pH} 7 .{ }^{48}$ Interestingly, Glu199 located near the catalytic triad was predicted to be neutral due to a high $\mathrm{p} K_{\mathrm{a}}$ value (ca. 10) for all the $\mathrm{AChE}$ and $\mathrm{BuChE}$ structures. Recently, Wan et al..$^{49}$ proposed that the protonated form of Glu199 can interact with a conserved water and stabilize the catalytic triad in the molecular simulations of the BuChE-tacrine complex. The optimization of the hydrogen bond network between the protein and reference ligand was performed to adjust the hydrogen atoms' orientation, followed by energy minimization with fixed heavy atoms. Due to the large size of the bis(n)-lophine derivatives, the water molecules were removed from the binding site.

In this work, the docking experiments were performed with the molecular docking program Glide under the standard precision (SP) mode. ${ }^{50}$ We redocked the reference ligands (i.e., the co-crystallized compounds) into their respective $\mathrm{AChE}$ and $\mathrm{BuChE}$ conformations to validate the docking protocol adopted herein. We selected the BuChE structure complexed with a dual-binding site tacrinetryptophan hybrid (Protein Data Bank (PDB) ID 6I0C from Homo sapiens, solved at $2.67 \AA$ ). ${ }^{51}$ For AChE, we selected four representative conformations of $\mathrm{AChE}$ to consider the significant conformational changes mainly observed on the PAS using an ensemble docking strategy. ${ }^{52,53}$ This approach consists of docking the compounds into each representative conformation of the receptor, aiming to implicitly consider large-scale protein movements. ${ }^{54} \mathrm{We}$ kept the conserved water molecules already reported in our previous work ${ }^{40}$ except for Wat720 (numbering from PDB code 6I0C for the BuChE structure), which is more exposed to the solvent and interacts with only one amino acid residue. For each ligand, the top-energy pose was selected according to the lowest Emodel value (i.e., the Emodel is the Glide scoring function recommended to evaluate different poses of the same ligand). Thus, the binding mode with the lowest GlideScore among the four AChE representative structures was selected for each compound. The protein conformations selected in this work for AChE were 1ZGC (Torpedo californica solved at 2.1 $\mathrm{A}$ ) (Haviv et al.), ${ }^{55}$ 2CKM (Torpedo californica solved at $2.1 \AA$ ), 1Q84 (Mus musculus, solved at $2.4 \AA$ ), ${ }^{56}$ and 4EY7 (Homo sapiens, solved at $2.4 \AA$ ). ${ }^{57}$ All the inhibitors from the four representative conformations of AChE act as dual inhibitors interacting with both CAS and PAS.

\section{Results and Discussion}

\section{Synthesis}

The symmetrical bis $(n)$-lophine analogues were synthesized through a one-pot four component reaction of pyridinecarboxaldehyde (1a-1c), 1,n-alkanediamines (2a-2c), benzil (3) and ammonium acetate (4, Scheme 1). The reaction was performed in a microwave reactor at $110{ }^{\circ} \mathrm{C}$ for $5 \mathrm{~h}$. The reaction provides the bis $(n)$-lophines analogues (5-7), in 31-55\% yield, in one reaction step.

For the synthesis of unsymmetrical bis $(n)$-lophine analogues, it was necessary synthesize the $N$-alkylaminolophine precursors $(9 a-9 c)$ through the multicomponent reaction between benzaldehyde $(\mathbf{8})$, 1,n-alkanediamines (2a-2c), benzil (3) and ammonium acetate (4, Scheme 2). In order to optimize the synthesis of precursors 9a-9c using a microwave reactor, we chose hexanediamine as substrate. The aim was to examine some of this reaction's general features, such as Lewis acid catalysts, stoichiometry, reaction time, temperature and reaction yield. 
<smiles></smiles><smiles>O=C(C(=O)c1ccccc1)c1ccccc1</smiles>

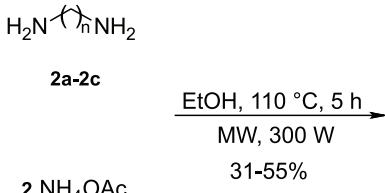

4

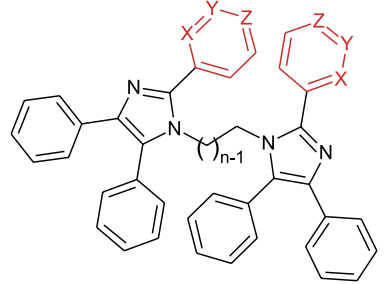

5a, $n=6, X=\mathrm{N}, \mathrm{Y}=\mathrm{CH}, Z=\mathrm{CH} \quad 6 a, n=7, X=\mathrm{N}, \mathrm{Y}=\mathrm{CH}, Z=\mathrm{CH}$ $\begin{array}{ll}\mathbf{5 a}, n=6, X=\mathrm{N}, \mathrm{Y}=\mathrm{CH}, Z=\mathrm{CH} & \mathbf{6 a}, \mathrm{n}=7, X=\mathrm{N}, \mathrm{Y}=\mathrm{CH}, Z=\mathrm{CH} \\ \mathbf{5 b}, \mathrm{n}=6, X=\mathrm{CH}, \mathrm{Y=N}, Z=\mathrm{CH} & \mathbf{6 b}, \mathrm{n}=7, X=\mathrm{CH}, \mathrm{Y=N}, Z=\mathrm{CH}\end{array}$ $\begin{array}{ll}\mathbf{5 c}, \mathrm{n}=6, X=\mathrm{CH}, \mathrm{Y}=\mathrm{CH}, \mathrm{Z}=\mathrm{N} & \mathbf{6 c}, \mathrm{n}=7, X=\mathrm{CH}, \mathrm{Y}=\mathrm{CH}, \mathrm{Z}=\mathrm{N}\end{array}$ $7 a, n=8, X=N, Y=C H, Z=C H$ $7 b, n=8, X=C H, Y=N, Z=C H$
$7 c, n=8, X=C H, Y=C H, Z=N$

Scheme 1. Synthesis of symmetrical bis(n)-lophine analogues.

As show in Table 1 (entry 6), the use of 1 equiv. of $\mathrm{NH}_{4} \mathrm{OAc}$ and 1 equiv. of hexanediamine, $110^{\circ} \mathrm{C}$ for $5 \mathrm{~h}$ and $\mathrm{InCl}_{3}$ as catalyst, afforded $9 \mathrm{a}$ in $50 \%$ yield. Interestingly, we found that in this reaction conditions, the use of Lewis acid was not necessary, affording the precursor 9a in 47\% yield (entry 10). In this way, the conditions of entry 10 were applied for the synthesis of $N$-alkylaminolophine precursors $\mathbf{9 b}$ and $9 \mathbf{c}$. Next, the multicomponent reaction of $9 \mathbf{a}-9 \mathbf{c}$ with pyridinecarboxaldehyde (1a-1c), benzil (3) and ammonium acetate (4) provided the unsymmetrical bis $(n)$-lophine analogues in 23-49\% yield (10-12, Scheme 3$)$.

\section{In vitro inhibition studies on AChE and BuChE}

$\mathrm{AChE}$ and $\mathrm{BuChE}$ activities were measured in vitro by the spectrophotometric method developed by Ellman et al., ${ }^{41}$ with slight modifications, with tacrine as the reference inhibitor. The inhibitors' effectiveness is expressed as $\mathrm{IC}_{50}$, representing the concentration of an inhibitor required for $50 \%$ inhibition of the enzyme. For this study, compounds with $\mathrm{IC}_{50}$ values over $50 \mu \mathrm{M}$ were considered to be inactive. All the compounds, except $\mathbf{7 a}, \mathbf{7 b}$ and $\mathbf{7 c}$, displayed potent inhibitory activity against $\mathrm{BuChE}$ at a micromolar and sub-micromolar range $\left(\mathrm{IC}_{50} 32.25-0.03 \mu \mathrm{M}\right)$. The inhibition was found to be highly selective since none of the bis $(n)$-lophine analogues were active against AChE, at the tested concentrations. BuChE inhibitory activity results are summarized in Table 2. These results indicate that BuChE inhibition is mainly influenced by two features: the R substituent nature and the length of the alkyl spacer. Among all the tested compounds, the asymmetric analogues of bis $(n)$-lophine with 2-pyridine and 3-pyridine moiety were better inhibitors of $\mathrm{BuChE}$ than those containing 4-pyridine moiety. Compound 11b, characterized by an unsubstituted<smiles>O=Cc1ccccc1</smiles>

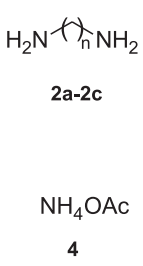

Scheme 2. Synthesis of $N$-alkylaminolophine precursors.

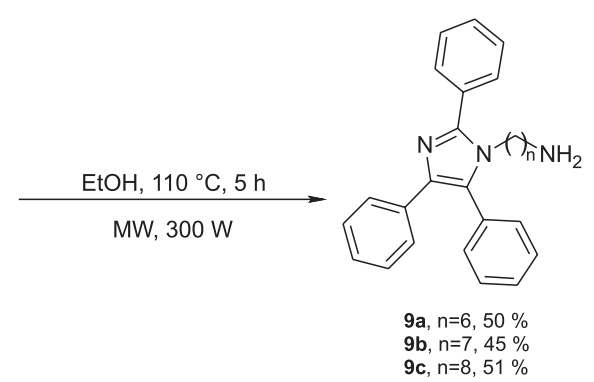

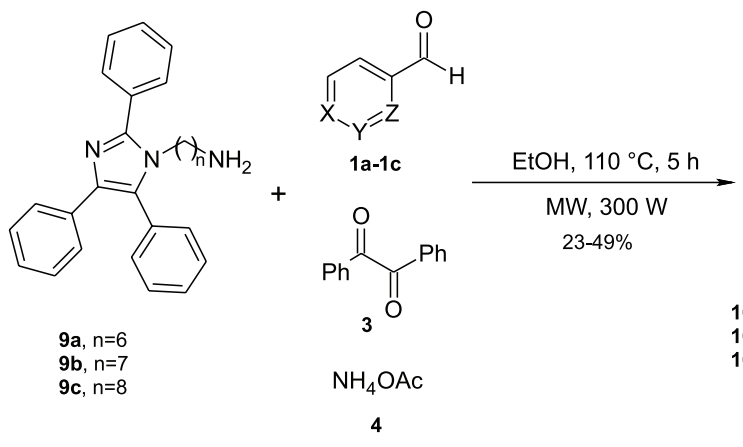

4

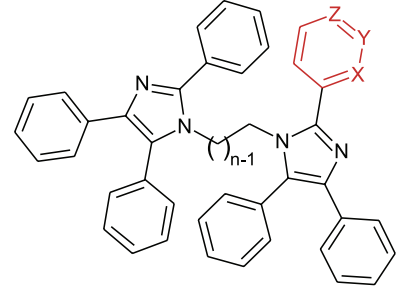

10a, $n=6, X=N, Y=C H, Z=C H \quad 11 a, n=7, X=N, Y=C H, Z=C H$

ob, $n=6, X=C H, Y=N, Z=C H \quad 11 b, n=7, X=C H, Y=N, Z=C H$

10c, $n=6, X=C H, Y=C H, Z=N \quad 11 c, n=7, X=C H, Y=C H, Z=N$

12a, $n=8, X=N, Y=C H, Z=C H$ 12b, $n=8, X=C H, Y=N, Z=C H$ 12c, $n=8, X=C H, Y=C H, Z=N$

Scheme 3. Synthesis of unsymmetrical bis $(n)$-lophine analogues. 
Table 1. One-pot four component reaction for the synthesis of $\mathbf{9 a}$ under different conditions

\begin{tabular}{|c|c|c|c|c|c|c|}
\hline entry & $\mathrm{NH}_{4} \mathrm{OAc} / \mathrm{mmol}$ & Hexanediamine / mmol & Temperature $/{ }^{\circ} \mathrm{C}$ & Catalyst $(0.15 \mathrm{~mol} \%)$ & time / h & Yield /\% \\
\hline 1 & 1 & 1 & 78 & $\mathrm{InCl}_{3}$ & 1 & 13 \\
\hline 2 & 1 & 2 & 78 & $\mathrm{InCl}_{3}$ & 1 & 8 \\
\hline 3 & 2 & 1 & 78 & $\mathrm{InCl}_{3}$ & 1 & 0 \\
\hline 4 & 1 & 1 & 110 & $\mathrm{InCl}_{3}$ & 3 & 40 \\
\hline 5 & 1 & 2 & 110 & $\mathrm{InCl}_{3}$ & 3 & 10 \\
\hline 6 & 1 & 1 & 110 & $\mathrm{InCl}_{3}$ & 5 & 50 \\
\hline 7 & 1 & 1 & 110 & $\mathrm{FeCl}_{3} \cdot 6 \mathrm{H}_{2} \mathrm{O}$ & 5 & 41 \\
\hline 8 & 1 & 1 & 110 & $\mathrm{CeCl}_{3}$ & 5 & 44 \\
\hline 9 & 1 & 1 & 110 & $\mathrm{SnCl}_{2} \cdot 2 \mathrm{H}_{2} \mathrm{O}$ & 5 & 34 \\
\hline 10 & 1 & 1 & 110 & - & 5 & 47 \\
\hline
\end{tabular}

Table 2. Inhibition of BuChE activity for bis(n)-lophine analogues

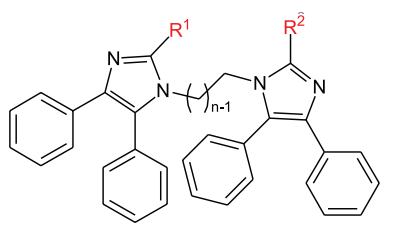

\begin{tabular}{|c|c|c|c|c|c|c|}
\hline entry & Compound & $\mathrm{R}^{1}$ & $\mathrm{R}^{2}$ & $\mathrm{n}$ & $\begin{array}{c}\mathrm{IC}_{50} \\
\mathrm{BuChE} / \\
\mu \mathrm{M}\end{array}$ & $\log \mathrm{IC}_{50} \pm \mathrm{SD}$ \\
\hline 1 & $5 a$ & 2-Py & 2-Py & 6 & 0.246 & $-0.608 \pm 0.062$ \\
\hline 2 & $5 \mathbf{b}$ & 3-Py & 3-Py & 6 & 0.208 & $-0.691 \pm 0.198$ \\
\hline 3 & $5 c$ & 4-Py & 4-Py & 6 & 25.84 & $1.412 \pm 0.053$ \\
\hline 4 & $6 a$ & 2-Py & 2-Py & 7 & 32.25 & $1.508 \pm 0.111$ \\
\hline 5 & $6 \mathbf{b}$ & 3-Py & 3-Py & 7 & 12.04 & $1.081 \pm 0.091$ \\
\hline 6 & $6 c$ & 4-Py & 4-Py & 7 & 23.27 & $1.367 \pm 0.125$ \\
\hline 7 & $7 a$ & 2-Py & 2-Py & 8 & n.a. & - \\
\hline 8 & $7 b$ & 3-Py & 3-Py & 8 & n.a. & - \\
\hline 9 & $7 \mathrm{c}$ & 4-Py & 4-Py & 8 & n.a. & - \\
\hline 10 & $10 a$ & $\mathrm{Ph}$ & 2-Py & 6 & 0.155 & $-0.808 \pm 0.058$ \\
\hline 11 & $10 \mathrm{~b}$ & $\mathrm{Ph}$ & 3-Py & 6 & 0.134 & $-0.874 \pm 0.075$ \\
\hline 12 & $10 \mathrm{c}$ & $\mathrm{Ph}$ & 4-Py & 6 & 0.164 & $-0.784 \pm 0.109$ \\
\hline 13 & 11a & $\mathrm{Ph}$ & 2-Py & 7 & 0.091 & $-1.041 \pm 0.039$ \\
\hline 14 & 11b & $\mathrm{Ph}$ & 3-Py & 7 & 0.034 & $-1.463 \pm 0.063$ \\
\hline 15 & $11 \mathrm{c}$ & $\mathrm{Ph}$ & 4-Py & 7 & 1.294 & $0.112 \pm 0.164$ \\
\hline 16 & $12 a$ & $\mathrm{Ph}$ & 2-Py & 8 & 0.578 & $-0.238 \pm 0.070$ \\
\hline 17 & $12 \mathrm{~b}$ & $\mathrm{Ph}$ & 3-Py & 8 & 0.071 & $-1.151 \pm 0.100$ \\
\hline 18 & $12 \mathrm{c}$ & $\mathrm{Ph}$ & 4-Py & 8 & 1.586 & $0.200 \pm 0.044$ \\
\hline 19 & tacrine & & & & 0.004 & $-2.35 \pm 0.07$ \\
\hline
\end{tabular}

$\mathrm{n}$ : number of methylenes in the linker; $\mathrm{IC}_{50}$ : half maximal inhibitory concentration; BuChE: butyrylcholinesterase; SD: standard deviation; Py: pyridine; Ph: phenyl; n.a.: not active.

phenyl ring, a seven-carbon spacer, and a 3-pyridine moiety, showed the most potent enzyme inhibition with an $\mathrm{IC}_{50}$ value of $0.034 \mu \mathrm{M}$. In the case of asymmetric analogues of bis(n)-lophine with an eight-carbon linker, the most active compound was 12b $\left(\mathrm{IC}_{50} 0.071 \mu \mathrm{M}\right)$, and for those with a spacer of six methylenes, compound $10 \mathrm{~b}$ resulted in the most active one $\left(\mathrm{IC}_{50} 0.134 \mu \mathrm{M}\right)$. Both derivatives $\mathbf{1 2 b}$ and 10b share with 11b the presence of phenyl and 3-pyridine moieties as $\mathrm{R}^{1}$ and $\mathrm{R}^{2}$. Since compound $\mathbf{1 1} \mathrm{b}$ was the most effective BuChE inhibitor of the series, it was selected for the kinetic study of enzyme inhibition.

\section{Kinetic characterization of BuChE inhibition}

Enzyme activity was evaluated at different fixed substrate concentrations and increasing inhibitor concentrations. The data were used to elucidate the enzyme inhibition mechanism, and the results are illustrated in the form of LineweaverBurk plots (Figure 1). The double reciprocal plots obtained using increasing inhibitors concentrations demonstrated that compound 11b behaves as a non-competitive inhibitor (see Figure 1). In fact, experimental data obtained using increasing inhibitor concentrations describe straight lines

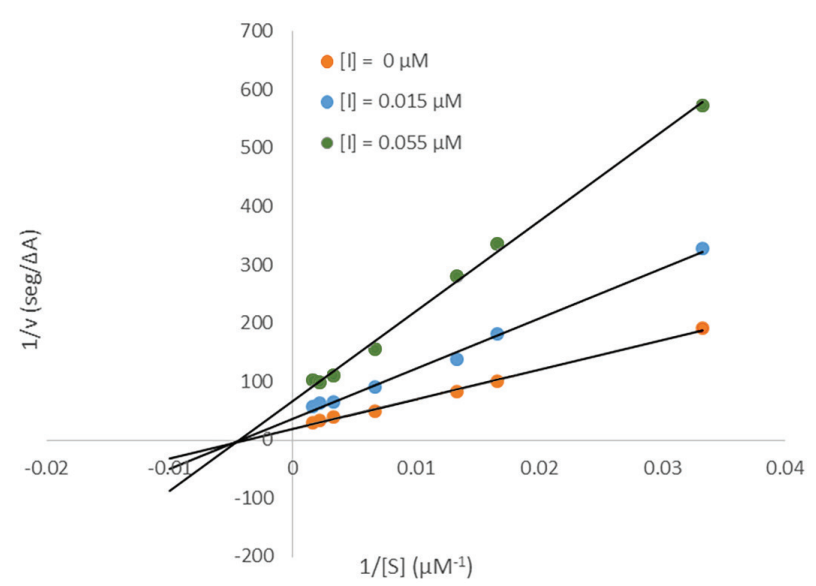

Figure 1. Lineweaver-Burk plots of the BuChE inhibition by compound 11b with butyrilthiocholine (S) as substrate. 
intersecting each other at a point on the $x$-axis. Moreover, the tested compounds determine the decrease of the maximum reaction velocity $\left(\mathrm{V}_{\max }\right)$, without affecting the MichaelisMenten constant $\left(\mathrm{K}_{\mathrm{m}}\right)$ values appreciably. Thus, the enzyme kinetic study suggests that the inhibitor binds to both the CAS and PAS sites of BuChE. The inhibition constant $\mathrm{Ki}$ was equal to $0.021 \mu \mathrm{M}$. For comparison purpose, the reported Ki of tacrine is about $0.017 \mu \mathrm{M}^{58}$

\section{Evaluation of cytotoxicity in in vitro models}

The bis $(n)$-lophine analogues $\mathbf{5 b}$ and $\mathbf{1 2 b}$ were evaluated for their cytotoxicity against cellular models of Vero (kidney), HepG2 (hepatic), and C6 (astroglial) cell lines, which are widely used as experimental models for seeking information about nephrotoxicity, hepatotoxicity, and neurotoxicity, respectively.

According to the results (see Table 3, and Supplementary Information section, Figures S38-S43), the compounds $\mathbf{5 b}$ and 12b showed no or slightly cytotoxic effects in all cellular models at the tested concentrations, exhibiting an $\mathrm{IC}_{50}$ higher than $125 \mu \mathrm{M}$. These results suggest that the compounds have a degree of safety because there is a large difference between the $\mathrm{IC}_{50}$ for $\mathrm{BuChE}$ inhibition $\left(\mathrm{IC}_{50}=0.208 \mu \mathrm{M}\right.$ for $\mathbf{5 b}$ and $0.071 \mu \mathrm{M}$ for $\mathbf{1 2 b}$ ) and the concentration to which all cell lines were exposed (up to $125 \mu \mathrm{M}$ ) in the cytotoxic assay. For the $\mathbf{5 b}$ and $\mathbf{1 2 b}$ derivatives, concentrations 600 and 1760 times higher than those active for inhibiting BuChE were used, respectively, and small or no reduction in the cell viability was observed. Also, it can be observed that the bis $(n)$-lophine analogues are significantly less toxic than tacrine to the hepatic cell line (HepG2), once at $125 \mu \mathrm{M}$ tacrine displayed a cell viability of $44 \%$, while the derivatives $\mathbf{5 b}$ and $\mathbf{1 2 b}$ showed about 70 and $95 \%$ in cell viability at the same concentration.

Table 3. Cell viability after treatment with the selected compounds for $24 \mathrm{~h}$ using MTT assay $\left(\mathrm{IC}_{50}\right)$ in Vero, HepG2 and C6 cell lines

\begin{tabular}{lccc}
\hline \multicolumn{4}{c}{ Cytotoxicity $\left(\mathrm{IC}_{50} \pm \mathrm{SEM}\right) / \mu \mathrm{M}$} \\
\hline Compound & Vero & HepG2 & C6 \\
\hline $\mathbf{5 b}$ & $>125$ & $>125$ & $>125$ \\
$\mathbf{1 2 b}$ & $>125$ & $>125$ & $>125$ \\
Tacrine & $168.9 \pm 14.0$ & $114.9+17.9$ & $450.7 \pm 4.2$ \\
\hline
\end{tabular}

$\mathrm{IC}_{50}$ : half maximal inhibitory concentration; SEM: standard error of the mean.

\section{Molecular docking}

The redocking experiment with the BuChE conformation used in this work (PDB code 6I0C) was able to reproduce the experimental binding mode of the co-crystallized ligand as the top-scored pose (GlideScore $=-11.022 \mathrm{kcal} \mathrm{mol}^{-1}$ ) within a root mean square deviation (RMSD) value of $0.53 \AA$ (Figure 2). The chlorotacrine moiety was perfectly predicted near CAS, whereas the indole group exhibited an inverted mode, making T-stacking interaction with W231, a residue involved in the selectivity against BuChE over AChE. Also, the ensemble docking strategy adopted for AChE was successfully validated with all the reference ligands docked with $\mathrm{RMSD} \leq 2 \AA$ in the respective protein conformation according to the lowest GlideScore. ${ }^{28}$

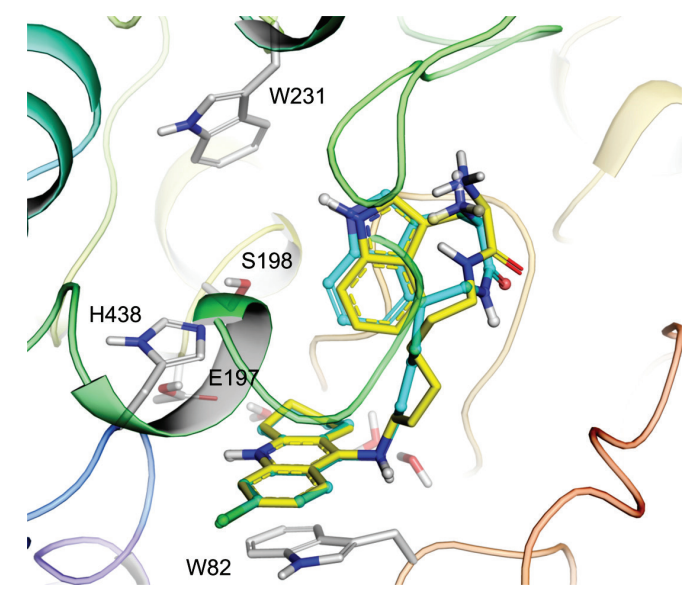

Figure 2. Redocking of the co-crystallized ligand of BuChE in the conformation with PDB code 6I0C (carbons in cyan) superimposed with the experimental binding mode (carbons in yellow).

The two most potent compounds $\mathbf{1 1 b}$ and 12b were ranked at the top-3 best scored ligands (Table 4, -11.28 and $-11.052 \mathrm{kcal} \mathrm{mol}^{-1}$, respectively) and predicted to interact with the BuChE cavity with similar binding modes, with the unsubstituted lophine moiety interacting at the bottom of the gorge, whereas the $N$-substituted lophine moiety interacts with the PAS region. At the bottom of the binding cavity, these ligands interact through lipophilic and T-stacking interactions, mainly with the side chains of key aromatic residues such as Trp82, His438, Trp321, Phe329, and Tyr332 (Figure 3). Despite these interactions, the conserved $\pi$-stacking or cation- $\pi$ interactions commonly observed with $\operatorname{Trp} 82$ for potent inhibitors and tacrine were not observed in the docking experiments, probably due to the larger size and small flexibility of the lophine moiety leading to a suboptimal fit to the binding site. Notably, the bis(n)-lophine derivatives are able to interact more deeply on the BuChE binding site than the AChE cavity, being closer to Trp82 in the first. For instance, a phenyl ring of the compound 11b was predicted to be within a distance of 3.5 and $5.5 \AA$ to the closest indole ring atom from Trp 82 of $\mathrm{BuChE}$ (GlideScore $=-11.28 \mathrm{kcal} \mathrm{mol}^{-1}$ ) and $\mathrm{AChE}$ 
Table 4. Docking results of the lophine derivatives and the reference compound tacrine against $\mathrm{BuChE}$ (PDB code 6I0C)

\begin{tabular}{lccc}
\hline entry & Compound & $\begin{array}{c}\text { GlideScore / } \\
\left(\mathrm{kcal} \mathrm{mol}^{-1}\right)\end{array}$ & $\mathrm{IC}_{50} / \mu \mathrm{M}$ \\
\hline 1 & $\mathbf{5 a}$ & -9.708 & 0.246 \\
2 & $\mathbf{5 b}$ & -9.657 & 0.208 \\
3 & $\mathbf{5 c}$ & -10.379 & 25.84 \\
4 & $\mathbf{6 a}$ & -10.808 & 32.25 \\
5 & $\mathbf{6 b}$ & -9.827 & 12.04 \\
6 & $\mathbf{6 c}$ & -10.468 & 23.27 \\
7 & $\mathbf{7 a}$ & -10.395 & n.a. \\
8 & $\mathbf{7 b}$ & -10.594 & n.a. \\
9 & $\mathbf{7 c}$ & -10.484 & n.a. \\
10 & $\mathbf{1 0 a}$ & -10.102 & 0.155 \\
11 & $\mathbf{1 0 b}$ & -9.98 & 0.134 \\
12 & $\mathbf{1 0 c}$ & -10.028 & 0.164 \\
13 & $\mathbf{1 1 a}$ & -10.405 & 0.091 \\
14 & $\mathbf{1 1 b}$ & -11.28 & 0.034 \\
15 & $\mathbf{1 1 c}$ & -11.323 & 1.294 \\
16 & $\mathbf{1 2 a}$ & -10.944 & 0.578 \\
17 & $\mathbf{1 2 b}$ & -11.052 & 0.071 \\
18 & $\mathbf{1 2 c}$ & -10.927 & 1.586 \\
19 & tacrine & $-7.578(0.934)^{\mathrm{a}}$ & 0.004 \\
\hline
\end{tabular}

${ }^{\mathrm{a}}$ Root mean square deviation from the experimental binding mode present in the 4BDS (PDB code for human BuChE in complex with tacrine) conformation of BuChE. $\mathrm{IC}_{50}$ : half maximal inhibitory concentration; n.a.: not active.

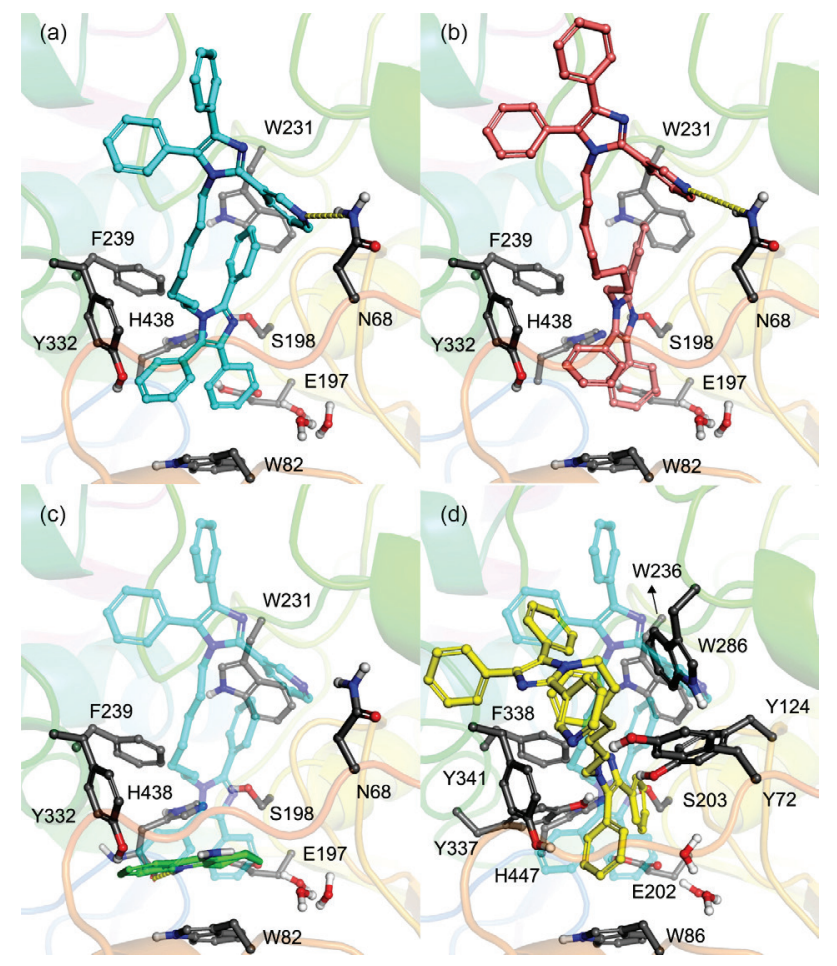

Figure 3. Predicted binding modes of the compounds (a) 11b (carbon atoms colored cyan), (b) 12b (carbon atoms colored cyan), and (c) tacrine (carbon atoms colored green) superimposed to $\mathbf{1 1 b}$ against $\mathrm{BuChE}$ (PDB code 6I0C). (d) Predicted binding mode of the compound 11b against $\mathrm{AChE}$ (carbon atoms colored yellow, PDB code 1Q84) superimposed with its binding mode predicted against $\mathrm{BuChE}$ (transparent sticks with carbon atoms colored cyan, PDB code 1Q84). Hydrogen bonds are represented as yellow dashed lines. (conformation 1Q84, GlideScore $=-9.234 \mathrm{kcal} \mathrm{mol}^{-1}$ ), respectively. In addition, the remarkable selectivity of the lophine dimers for BuChE over AChE might also be due to the absence of some aromatic residues at the $\mathrm{BuChE}$ binding site, leading to an opened binding site that is able to best accommodate such large ligands and the formation of specific hydrophobic pockets frequently related with selectivity against BuChE. For example, according to the docking results, the bis $(n)$-lophine dimers interacted with the Trp231 side chain through a T-stacking interaction, a residue only accessible in $\mathrm{BuChE}$ and claimed to be a key residue for selectivity. ${ }^{40,59}$

Despite the predicted binding affinities being quite similar for all compounds, probably the main difference in the experimental affinities observed between the series of homo and heterodimers is related to the desolvation penalty arising from burying the pyridine group in the homodimer series without compensation with polar interactions with the environment. According to the docking results, all heterodimer derivatives were predicted to have the unsubstituted lophine moiety interacting at the bottom of the cavity, except the compounds with the nitrogen atom at the ortho position. On the other hand, the $N$-substituted phenyl ring at meta-position of the heterodimer compounds, such as $\mathbf{1 0 b}$ and $\mathbf{1 1 b}$, allowed the formation of a hydrogen bond with the Asn68 side chain from PAS (2.92 and $3.17 \AA$, respectively).

\section{Conclusions}

In summary, two new series of bis(n)-lophine analogues were synthesized through a one-pot four component reaction performed in a microwave reactor. Also, a new protocol for the synthesis of $\mathrm{N}$-alkylaminolophine precursors was developed without the need of Lewis acid as catalyst. The bis(n)-lophine analogues were strongly selective to $\mathrm{BuChE}$ and present $\mathrm{IC}_{50}$ at a micromolar and sub-micromolar range $\left(\mathrm{IC}_{50} 32.25-0.03 \mu \mathrm{M}\right)$. Compounds $\mathbf{1 1 b}\left(\mathrm{IC}_{50}=0.034 \mu \mathrm{M}\right)$ and $\mathbf{1 2 b}\left(\mathrm{IC}_{50}=0.071 \mu \mathrm{M}\right)$ were the most potent inhibitors of the asymmetric bis $(n)$-lophines series. Of the symmetric bis $(n)$-lophines, the most active compound was $\mathbf{5 b}$ $\left(\mathrm{IC}_{50}=0.208 \mu \mathrm{M}\right)$. The kinetic characterization of BuChE inhibition by compound $\mathbf{1 1 b}$ suggests that the compound bind to both CAS and PAS sites, corroborated by the docking results. According to the predicted binding mode for the compound $\mathbf{1 1 b}$, it is able to interact deeply into the BuChE binding site and a hydrophobic pocket involved with selectivity formed mainly by the residue Trp231, which is only available at the BuChE cavity. The dimers 5b and 12b showed no cytotoxic effects in Vero (renal), HepG2 (hepatic), and C6 (astroglial) cell lines and a wide 
range of safety considering the active concentration for BuChE inhibition.

\section{Supplementary Information}

Supplementary data $\left({ }^{1} \mathrm{H}\right.$ and ${ }^{13} \mathrm{C}$ NMR spectra and cytotoxicity results) are available free of charge at http://jbcs.sbq.org.br as PDF file.

\section{Acknowledgments}

We would like to thank the following Brazilian and Argentine agencies for financial support and fellowships: CNPq, FAPERGS, CAPES (finance code 001), PROPESQUFRGS, LNCC, CONICET, ANPCyT, UNS and AUGM.

\section{Author Contributions}

Viktor S. Câmara was responsible for the data curation, investigation, methodology, visualization, writing original draft, review and editing; Ana Julia Soares for data curation and methodology; Brunella Biscussi for data curation, formal analysis, investigation, methodology, visualization, writing original draft; Ana Paula Murray for the data curation, formal analysis, funding acquisition, investigation, methodology, resources, supervision and writing original draft; Isabella A. Guedes for the conceptualization, data curation, formal analysis, investigation, software, writing original draft; Laurent E. Dardenne for the conceptualization, data curation, funding acquisition, investigation, software, supervision and writing original draft; Thais C. Ruaro for the data curation, formal analysis, investigation, methodology, validation and writing original draft; Aline R. Zimmer for the data curation, formal analysis, funding acquisition, investigation, methodology, resources supervision and writing original draft; Marco A. Ceschi for the conceptualization, data curation, formal analysis, funding acquisition, investigation, methodology, project administration, resources, supervision, writing original draft, review and editing.

\section{References}

1. Sharma, K.; Mol. Med. Rep. 2019, 20, 1479.

2. Green, H.; Tsitsi, P.; Markaki, I.; Aarsland, D.; Svenningsson, P.; CNS Drugs 2019, 33, 143.

3. Li, Q.; Yang, H. Y.; Chen, Y.; Sun, H. P.; Eur. J. Med. Chem. 2017, 132, 294.

4. Darvesh, S.; Darvesh, K. V.; McDonald, R. S.; Mataija, D.; Walsh, R.; Mothana, S.; Lockridge, O.; Martin, E.; J. Med. Chem. 2008, 51, 4200.
5. Moodie, L. W. K.; Sepcic, K.; Turk, T.; Frangez, R.; Svenson, J.; Nat. Prod. Rep. 2019, 36, 1053.

6. McKeith, I.; del Ser, T.; Spano, P.; Emre, M.; Wesnes, K.; Anand, R.; Cicin-Sain, A.; Ferrara, R.; Spiegel, R.; Lancet 2000, 356, 2031.

7. Eldufani, J.; Blaise, G.; Alzheimer's Dementia: Transl. Res. Clin. Interventions 2019, 5, 175.

8. Kumar, A.; Singh, A.; Ekavali; Pharmacol. Rep. 2015, 67, 195.

9. Andrade, S.; Ramalho, M. J.; Loureiro, J. A.; Pereira, M. D.; Int. J. Mol. Sci. 2019, 20, 2313.

10. McEneny-King, A.; Osman, W.; Edginton, A. N.; Rao, P. P. N.; Bioorg. Med. Chem. Lett. 2017, 27, 2443.

11. Panza, F.; Lozupone, M.; Seripa, D.; Imbimbo, B. P.; Ann. Neurol. 2019, 85, 303.

12. Macdonald, I. R.; Maxwell, S. P.; Reid, G. A.; Cash, M. K.; Debay, D. R.; Darvesh, S.; J. Alzheimer's Dis. 2017, 58, 491.

13. Greig, N. H.; Lahiri, D. K.; Sambamurti, K.; Int. Psychoger. 2002, 14, 77 .

14. Liu, S. Y.; Xiong, H.; Yang, J. Q.; Yang, S. H.; Li, Y. F.; Yang, W. C.; Yang, G. F.; ACS Sens. 2018, 3, 2118.

15. Chen, V. P.; Gao, Y.; Geng, L. Y.; Parks, R. J.; Pang, Y. P.; Brimijoin, S.; Proc. Natl. Acad. Sci. U. S. A. 2015, 112, 2251.

16. Taylor, P.; Radic, Z.; Annu. Rev. Pharmacol. Toxicol. 1994, 34 , 281.

17. Qiu, G. L.; He, S. S.; Chen, S. C.; Li, B.; Wu, H. H.; Zhang, J.; Tang, W. J.; J. Enzyme Inhib. Med. Chem. 2018, 33, 1506.

18. Darvesh, S.; Macdonald, I. R.; Martin, E.; Bioorg. Med. Chem. Lett. 2013, 23, 3822.

19. Darvesh, S.; Curr. Alzheimer Res. 2016, 13, 1173.

20. Guillozet, A. L.; Smiley, J. F.; Mash, D. C.; Mesulam, M. M.; Ann. Neurol. 1997, 42, 909.

21. Saxena, A.; Redman, A. M. G.; Jiang, X. L.; Lockridge, O.; Doctor, B. P.; Biochemistry 1997, 36, 14642.

22. Masson, P.; Carletti, E.; Nachon, F.; Protein Pept. Lett. 2009, 16, 1215.

23. Pang, Y. P.; Quiram, P.; Jelacic, T.; Hong, F.; Brimijoin, S.; J. Biol. Chem. 1996, 271, 23646.

24. Sameem, B.; Saeedi, M.; Mahdavi, M.; Shafiee, A.; Eur. J. Med. Chem. 2017, 128, 332.

25. Milelli, A.; de Simone, A.; Ticchi, N.; Chen, H. H.; Betari, N.; Andrisano, V.; Tumiatti, V.; Curr. Med. Chem. 2017, 24, 3522.

26. Korabecny, J.; Spilovska, K.; Mezeiova, E.; Benek, O.; Juza, R.; Kaping, D.; Soukup, O.; Curr. Med. Chem. 2019, 26, 5625.

27. Mesiti, F.; Chavarria, D.; Gaspar, A.; Alcaro, S.; Borges, F.; Eur. J. Med. Chem. 2019, 181, 111572.

28. Ceschi, M. A.; Pilotti, R. M.; Lopes, J. P. B.; Dapont, H.; da Rocha, J. B. T.; Afolabi, B. A.; Guedes, I. A.; Dardenne, L. E.; J. Braz. Chem. Soc. 2020, 31, 857.

29. Viegas, F. P. D.; Silva, M. F.; da Rocha, M. D.; Castelli, M. R.; Riquiel, M. M.; Machado, R. P.; Vaz, S. M.; de Lima, L. M. S.; Mancini, K. C.; de Oliveira, P. C. M.; Morais, E. P.; Gontijo, 
V. S.; da Silva, F. M. R.; Pecanha, D. D. A. F.; Castro, N. G.; Neves, G. A.; Giusti-Paiva, A.; Vilela, F. C.; Orlandi, L.; Camps, I.; Veloso, M. P.; Coelho, L. F. L.; Ionta, M.; Ferreira-Silva, G. A.; Pereira, R. M.; Dardenne, L. E.; Guedes, I. A.; Carneiro Jr., W. O.; Bellozi, P. M. Q.; de Oliveira, A. C. P.; Ferreira, F. F.; Pruccoli, L.; Tarozzi, A.; Viegas Jr., C.; Eur. J. Med. Chem. 2018, 147, 48 .

30. Fridman, N.; Kaftory, M.; Speiser, S.; Sens. Actuators, B 2007, 126, 107

31. Cardoso, A. L.; Lemos, A.; Melo, T.; Eur. J. Org. Chem. 2014, 5159.

32. Nakashima, K.; Biomed. Chromatogr. 2003, 17, 83.

33. Rossi, R.; Angelici, G.; Casotti, G.; Manzini, C.; Lessi, M.; Adv. Synth. Catal. 2019, 361, 2737.

34. Bansal, R.; Soni, P. K.; Ahirwar, M. K.; Halve, A. K.; Int. Res. J. Pure Appl. Chem. 2016, 11, DOI: 10.9734/ IRJPAC/2016/24493.

35. Heravi, M. M.; Daraie, M.; Zadsirjan, V.; Mol. Diversity 2015, 19, 577.

36. Lopes, J. P. B.; Camara, V. S.; Russowsky, D.; Santos, F. D.; Beal, R.; Nogara, P. A.; da Rocha, J. B. T.; Goncalves, P. F. B.; Rodembusch, F. S.; Ceschi, M. A.; New J. Chem. 2018, 42, 17126.

37. da Costa, J. S.; Lopes, J. P. B.; Russowsky, D.; Petzhold, C. L.; Borges, A. C. A.; Ceschi, M. A.; Konrath, E.; Batassini, C.; Lunardi, P. S.; Gonçalves, C. A. S.; Eur. J. Med. Chem. 2013, $62,556$.

38. Lopes, J. P. B.; da Costa, J. S.; Ceschi, M. A.; Gonçalves, C. A. S.; Konrath, E. L.; Karl, A. L. M.; Guedes, I. A.; Dardenne, L. E.; J. Braz. Chem. Soc. 2017, 28, 2218.

39. Ceschi, M. A.; da Costa, J. S.; Lopes, J. P. B.; Camara, V. S.; Campo, L. F.; Borges, A. C. D.; Gonçalves, C. A. S.; de Souza, D. F.; Konrath, E. L.; Karl, A. L. M.; Guedes, I. A.; Dardenne, L. E.; Eur. J. Med. Chem. 2016, 121, 758.

40. Lopes, J. P. B.; Silva, L.; Ceschi, M. A.; Ludtke, D. S.; Zimmer, A. R.; Ruaro, T. C.; Dantas, R. F.; de Salles, C. M. C.; Silva, F. P.; Senger, M. R.; Barbosa, G.; Lima, L. M.; Guedes, I. A.; Dardenne, L. E.; MedChemComm 2019, 10, 2089.

41. Ellman, G. L.; Courtney, K. D.; Andres, V.; Featherstone, R. M.; Biochem. Pharmacol. 1961, 7, 88.

42. GraphPad Prism, version 5.0; GraphPad Software Inc., San Diego, USA, 2007.

43. Mosmann, T.; J. Immunol. Methods 1983, 65, 55.

44. Maestro (Small-molecule Drug Discovery Suite 2018-4); Schrödinger, LLC, New York, NY, 2018.

45. Shelley, J. C.; Cholleti, A.; Frye, L. L.; Greenwood, J. R.; Timlin, M. R.; Uchimaya, M.; J. Comput.-Aided Mol. Des. 2007, 21, 681.
46. Greenwood, J. R.; Calkins, D.; Sullivan, A. P.; Shelley, J. C.; J. Comput.-Aided Mol. Des. 2010, 24, 591.

47. Sastry, G. M.; Adzhigirey, M.; Day, T.; Annabhimoju, R.; Sherman, W.; J. Comput.-Aided Mol. Des. 2013, 27, 221.

48. Olsson, M. H. M.; Sondergaard, C. R.; Rostkowski, M.; Jensen, J. H.; J. Chem. Theory Comput. 2011, 7, 525.

49. Wan, X.; Yao, Y.; Fang, L.; Liu, J. J.; Phys. Chem. Chem. Phys. 2018, 20, 14938.

50. Friesner, R. A.; Banks, J. L.; Murphy, R. B.; Halgren, T. A.; Klicic, J. J.; Mainz, D. T.; Repasky, M. P.; Knoll, E. H.; Shelley, M.; Perry, J. K.; Shaw, D. E.; Francis, P.; Shenkin, P. S.; J. Med. Chem. 2004, 47, 1739.

51. Chalupova, K.; Korabecny, J.; Bartolini, M.; Monti, B.; Lamba, D.; Caliandro, R.; Pesaresi, A.; Brazzolotto, X.; Gastellier, A. J.; Nachon, F.; Pejchal, J.; Jarosova, M.; Hepnarova, V.; Jun, D.; Hrabinova, M.; Dolezal, R.; Karasova, J. Z.; Mzik, M.; Kristofikova, Z.; Misik, J.; Muckova, L.; Jost, P.; Soukup, O.; Benkova, M.; Setnicka, V.; Habartova, L.; Chvojkova, M.; Kleteckova, L.; Vales, K.; Mezeiova, E.; Uliassi, E.; Valis, M.; Nepovimova, E.; Bolognesi, M. L.; Kuca, K.; Eur. J. Med. Chem. 2019, 168, 491.

52. Bourne, Y.; Taylor, P.; Radic, Z.; Marchot, P.; Embo J. 2003, 22,1 .

53. Johnson, G.; Moore, S. W.; Curr. Pharm. Des. 2006, 12, 217.

54. Guedes, A. I.; Magalhães, C. S.; Dardenne, L. E.; Biophys. Rev. 2014, 6, 75 .

55. Haviv, H.; Wong, D. M.; Greenblatt, H. M.; Carlier, P. R.; Pang, Y. P.; Silman, I.; Sussman, J. L.; J. Am. Chem. Soc. 2005, 127, 11029.

56. Bourne, Y.; Kolb, H. C.; Radic, Z.; Sharpless, K. B.; Taylor, P.; Marchot, P.; Proc. Natl. Acad. Sci. U. S. A. 2004, 101, 1449.

57. Cheung, J.; Rudolph, M. J.; Burshteyn, F.; Cassidy, M. S.; Gary, E. N.; Love, J.; Franklin, M. C.; Height, J. J.; J. Med. Chem. 2012, 55, 10282.

58. Roldan-Pena, J. M.; Romero-Real, V.; Hicke, J.; Maya, I.; Franconetti, A.; Lagunes, I.; Padron, J. M.; Petralla, S.; Poeta, E.; Naldi, M.; Bartolini, M.; Monti, B.; Bolognesi, M. L.; Lopez, O.; Fernandez-Bolanos, J. G.; Eur. J. Med. Chem. 2019, 181, 111550 .

59. Dighe, S. N.; Deora, G. S.; de la Mora, E.; Nachon, F.; Chan, S.; Parat, M. O.; Brazzolotto, X.; Ross, B. P.; J. Med. Chem. 2016, $59,7683$.
Submitted: November 30, 2020 Published online: February 8, 2021 